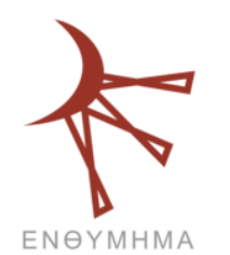

Enthymema XXVIII 2021

Durs Grünbein. Un'idea di Dante 3D

Rosalba Maletta

Università degli Studi di Milano

\begin{abstract}
Il saggio esamina alcuni contributi pubblicati dal poeta Durs Grünbein in occasione del settimo centenario della morte di Dante. Particolare attenzione viene dedicata alla terzina di Paradiso XXXIII, vv. 94-96 che impegna il poeta tedesco almeno dal 2009. In base alla terminologia impiegata da Grünbein per descrivere le sue trasposizioni dantesche in termini di rendering, il saggio indaga I'influenza del Sommo Fiorentino nella poetica dell'autore di Dresda alla luce dell'era globale-digitale per risalire alle fonti dell'ispirazione poetica, dove Grünbein rilegge Dante con Cartesio e Mandel'štàm.
\end{abstract}

Parole chiave - Grünbein; Dante; Rendering; Ispirazione poetica; Paragoni; Slittamenti metaforici; Era global-digitale.

Abstract - The essay examines some contributions published by the poet Durs Grünbein on the occasion of the seventh centenary of Dante's death. Particular attention is paid to the tercet of Paradiso XXXIII, 94-96, whose meaning and translation engage the German poet since at least 2009. On the basis of the terminology used by Grünbein to describe his transpositions of Dante's verses in terms of rendering, the essay investigates the influence of Dante in the author's poetics in the light of the global-digital era starting from the contributions of 2021 and going back to the sources of poetic inspiration, where Grünbein rereads Dante with Descartes and Mandelstam.

Keywords - Grünbein; Dante; Rendering; Poetic Inspiration; Comparisons; Metaphorical Shifts; Global-digital Era.

Maletta, Rosalba. "Durs Grünbein. Un'idea di Dante 3 D". Enthymema, n. XXVIII, 2021, pp. 171-203.

http://dx.doi.org/10.54103/2037-2426/16780

https://riviste.unimi.it/index.php/enthymema

(c) C)
(C)
ISSN 2037-2426 


\title{
Durs Grünbein. Un'idea di Dante 3D
}

\author{
Rosalba Maletta \\ Università degli Studi di Milano
}

\begin{abstract}
L'impressione genuina del postero, incontrandosi in Dante, non è d'imbattersi in un tenace e ben conservato sopravvissuto, ma di raggiungere qualcuno arrivato prima di lui. ${ }^{1}$

Non per foco, ma per divin'arte (Inferno XXI, v. 16) ${ }^{2}$

Ein wenig klebrig ist es, das Meer, milchweiß, sagt Michelet. Dann wäre Blau Illusion, die große neptunische Täuschung, Um das Weiß, schreckliche Wahrheit, erträglich zu machen. ${ }^{3}$
\end{abstract}

\section{Premessa}

Instancabile protagonista di una produzione poetica, saggistica e letteraria che non ha l'eguale nel panorama occidentale contemporaneo, Durs Grünbein omaggia Dante nel settimo centenario della morte con il trasporto e l'accomodamento nella propria lingua di una lirica giovanile - Guido, i' vorrei - e di una terzina dall'ultimo canto del Paradiso (XXXIII, vv. 94-96), ${ }^{4}$ di cui specialmente ci occuperemo.

Queste nuove prove su Dante si aggiungono a scritti precedenti, con l'intento dichiarato di mostrare quanto la presenza del "maestro" e "autore" (Inferno I, v. 85) sia per Grünbein di costante, comprovata contemporaneità: 5

${ }^{1}$ Contini, "Un'interpretazione di Dante." 111. Il titolo del presente contributo riecheggia Un'idea di Dante - silloge tuttora seminale e feconda che Gianfranco Contini diede alle stampe nel 1970.

${ }^{2}$ Per la Commedia citiamo da: Alighieri, Dante. La Divina Commedia. D'ora innanzi direttamente nel testo con Cantica, Canto e versi corrispondenti.

3 «Un po' viscoso è il mare, biancolatte, dice Michelet. / Blu sarebbe allora illusione, il grande inganno di Nettuno, /per rendere il bianco, spaventosa verità, sopportabile» (Grünbein, "Studien in Aquamarin." 108). Le traduzioni dai testi in lingua sono sempre a mia cura.

${ }^{4}$ Cito dal formato word gentilmente fornitomi dall'autore. Il testo "Ein flotter Sechser" è pubblicato in: Alighieri. 1 Sonett 30 Übersetzungen. La trasposizione e il commento del poeta sono il N. 10 delle 30 prove di traduzione e commento. Per la terzina di Paradiso XXXIII, vv. 94-96, tradotta e chiosata dal poeta, si veda: "DANTES VERSE". Trattasi di una Serie che la F.A.Z. ha riservato, nell'Anno di Dante, a artisti e pensatori per la cura di Jürgen Kaube: Grünbein, "Die unheimlichste Stelle."

5 Sulla presenza di Dante nel corpus grünbeiniano si rimanda ai seguenti contributi: Lampart (2002); Eskin (2002; 2010; 2013); Krämer (2007); Unfer Lukoschick-Dallapiazza (2011); Ruzzenenti (2018); Maletta (2021). Nel 2009, passando in rassegna la ricezione di Dante in area linguistica tedesca, Unfer Lukoschik rilevava come il nuovo millennio si fosse aperto con apporti interessanti, tra cui Grünbein che «compone poesie d'ispirazione dantesca (Der Soldat 1999)» (2009, 190). Su questo si veda pure il summenzionato Unfer Lukoschick; Dallapiazza (2011). Una straordinaria documentazione su come Grünbein legga e interpreti Dante è dato ricavare da due eventi fruibili in rete. In Una conversazione con Dante il 31/10/2020 alle ore 21.00 Durs Grünbein ha dialogato da Berlino con il germanista Maurizio Pirro. L'Incontro - promosso dalla Fondazione di Monte Verità e inizialmente concepito come una conversazione tra Durs Grünbein, Stefano Prandi e Fabio Pusterla, accompagnata da letture di Leonardo de 


\section{Durs Grünbein. Un'idea di Dante 3D \\ Rosalba Maletta}

D. G. - Riesig passt aber wie eine Wolkenschicht immer diese eine Leuchtturmgestalt hindurch - Dante. Das Magische darin, eine Art metaphysische Spekulation, ist, dass sich Dante selber so verstanden hat, es schien ihm bewußt zu sein, dass er diese Rolle in der Zukunft spielen würde. Das gibt es nicht so oft in der Kunst. Vielleicht hatte Leonardo Da Vinci ein ähnliches Gefühl, was er darstellen würde, gewisse Maler scheinen es auch gespürt zu haben, dass sie diese ,Transformatoren-Stellen' sind. Transformatoren ist vielleicht ein gutes Wort, weil sie im wörtlichen Sinne ihrerseits diese ganze translatio-Tradition übersetzen, die im Christentum, in der Theologie liegt. Dante ist aus dieser Sicht der Unvermeidliche. Man kommt nicht an ihm vorbei, und dann wird man mit Dante viele andere entdecken. Er ist der Systematiker, der sein Hauptwerk als eine Art Arche-Noah der Literatur erbaut und alle möglichen Schlüsselfiguren als Einzelexemplare aus der Texte-Flut rettet. (Grünbein; Ruzzenenti)

D. G. - Dante: questa figura-faro attraversa le epoche da gigante, vi si adatta come uno strato di nubi. La componente magica, una sorta di speculazione metafisica, è che Dante stesso si è visto così, pareva consapevole che in futuro avrebbe interpretato questo ruolo. Non capita spesso nell'arte. Forse Leonardo Da Vinci esperì un simile sentire su quanto intendeva rappresentare, certi pittori sembrano aver intuito di fungere da "transiti-trasformatori". Trasformatori è forse una designazione appropriata perché, a loro volta, alla lettera essi traghettano tutta questa tradizione di translatio che risiede nel cristianesimo, nella teologia. Da questo punto di vista Dante è l'Inevitabile. Non è dato oltrepassarlo, e con Dante se ne scoprono poi molti altri. Egli è il sistematico che costruisce la sua opera principale quasi fosse un'arca di Noè della letteratura salvando dal diluvio dei testi come esemplari individuali tutte le possibili figure chiave.

\section{Trans-formare}

Ne emerge un'idea di Dante nocchiero-traghettatore di una poetica capace di cogliere i mutamenti che dal sociale tornano al singolo individuo, quel poeta la cui dimensione personale è per Grünbein oggi di esule cosmopolita. ${ }^{6}$

Chi, come il poeta, per creare non ha che le parole, funge da trasformatore. Trasformatore elettrico, polo che converte tensione e potenza di versi e strofe nella matrice digitalizzata trasferendo filiazioni, genealogie e gemmazioni tràdite.

$\mathrm{Da}$ questo osservatorio e da questa posizione nel 2021 Grünbein traduce la terzina di $\mathrm{Pa}$ radiso XXXIII, vv. 94-96:

Colle - è stato sostituito da un dialogo-intervista in remoto a causa dell'emergenza sanitaria:http://www.fondazioneteatro.ch/it/eventiletterari/services/event.html?evid=64ad5e28-496c-4420 -a17a-366740496c11 (ultima consultazione: 18.08.2021). Sei anni prima, presso l'ICI Berlin (Kulturlabor Institute for Cultural Inquiry), precisamente il 27 febbraio 2014 alle ore 19.30, si è tenuto l'incontro Dichtung als Wissen. Durs Grünbein spricht über Dante. Durs Grünbein ha parlato del suo Dante con Manuele Gragnolati, Fabian Lampart e Aldo Venturelli: https://www.iciberlin.org/events/dichtung-als-wissen/ (ultima consultazione: 18.08.2021).

${ }^{6}$ Si veda la lirica Kosmopolit - dapprima apparsa in Nach den Satiren (1999, 85), indi riprodotta in Die Bars von Atlantis (2009, 7). Nella medesima raccolta di prose poetiche è contenuta, come settima "immersione", la prosa omonima Kosmopolit (31-33). La caratterizzazione torna in data primo gennaio 2000 in forma diaristica, allorché il poeta volge lo sguardo al decennio che ha attraversato dopo il suo approdo all'Ovest e una progressiva affermazione che lo ha condotto in vari luoghi del pianeta:«Seltsamer Rückblick auf ein Jahrzehnt der hektischen Globetrotterei und des kurzatmigen Reisens in die Hauptstädte Europas. Verspäteter Kosmopolit, so lange eingesperrt hinter Mauern, blieb dir nur der Schnellkurs. Im Eilverfahren desillusioniert» (Das erste Jahr 13) - «Singolare riepilogo di un decennio di vagabondaggi frenetici in giro per il mondo e brevi tappe nelle capitali europee. Cosmopolita in ritardo, così a lungo recluso dietro i muri, non ti rimaneva che un corso intensivo. Disilluso nella procedura d'urgenza». 


\section{Durs Grünbein. Un'idea di Dante 3D \\ Rosalba Maletta}

Un punto solo m'è maggior letargo

Che venticinque secoli a la 'impresa,

Che fé Nettuno ammirar l'ombra d'Argo. (Paradiso XXXIII, vv. 94-96)

Ein wenig Schlafsucht bringt mich mehr ins Arge

als zweieinhalb Jahrtausende zuvor der Schatten,

der Neptun staunen ließ: die Heldenfahrt der Argo. ${ }^{7}$

Nel commento che segue questa trasposizione, il poeta caratterizza la terzina come «die unheimlichste Stelle», con il significante unheimlich, su cui psicoanalisi e critica letteraria hanno versato fiumi di inchiostro, al superlativo assoluto. ${ }^{8} \mathrm{Il}$ passaggio riflette la prospettiva del dio, surclassato dall'umano progresso, e dell'umano che si predispone a contemplare il cosmo dalla prospettiva del poeta.

Dal tempo di Dante al tempo di Grünbein Nettuno non cessa di imbattersi nella natura umana, il mito non cessa di stupirsi dinanzi al tempo-freccia dello sviluppo tecnologico:

Man stelle sich einen Gott vor, der von den Unternehmungen der Menschheit (impresa) eines Tages überrumpelt wird. Als Taucher ist mir das Bild vertraut: der unheimliche Moment, wenn ein großer Schiffskörper die Wasserfläche schneidet und seinen Schatten wirft. Die Stelle ist etwas vieldeutig, bis heute erklärungsbedürftig. Meine Interpretation: Wenn Dante, der christliche Augenzeuge, nur einen Moment lang schwächelt und einschläft, gerät er leicht in die Position des alten Meergottes, den eine Erscheinung aufschreckt (etwa die Mond- und Marsmissionen unserer Gegenwart). Schon ist es zu spät und ein neues Zeitalter hat begonnen.("Die unheimlichste Stelle" - c. m.)

Immaginiamo un dio che un giorno sia colto alla sprovvista dalle imprese dell'umanità (impresa). Come subacqueo ho familiarità con l'immagine: il momento inquietante (unheimlich), in cui lo scafo di una grande nave taglia la superficie dell'acqua e proietta la sua propria ombra. Il passaggio è alquanto plurivoco, ancora oggi abbisogna di chiarimenti. Ecco la mia interpretazione: se Dante, il testimone oculare cristiano, si lascia andare solo un istante e si addormenta, non ci vuol molto perché si trovi nella posizione dell'antico dio del mare che viene scosso da un'apparizione (come le odierne missioni sulla luna e su Marte). È già troppo tardi e una nuova era è cominciata.

\section{Trans-letture}

Il metodo di lettura che intendiamo seguire per la nostra ricognizione intorno al «passaggio più unbeimlich» è quello che Grünbein propone per la propria opera e per gli autori che predilige in quanto lettore:

Als Leser streiche ich mir gern Stellen an, die ich mit anderen Stellen in anderen Büchern verknüpfen kann. So entsteht ein Gewebe aus Textpassagen, die zueinander passen, einander ergänzen und erweitern. Ein solches Vorgehen seitens des Lesers habe ich mir immer auch für meine Schriften gewünscht. Ich sehe mich als Teil eines Kontinuums zentraler Gedanken, an

7 Prima dei versi nel contributo della F.A.Z. leggiamo: «Ganz zum Schluss der Commedia lässt Dante aufblitzen, was der größte Schrecken für die Menschheit sein könnte: der Fortschritt" - «proprio alla fine della Commedia Dante fa balenare quel che per l'umanità potrebbe essere il maggior terrore: il progresso" ("Die unheimlichste Stelle").

${ }^{8}$ Non è possibile e neppure opportuno, nel contesto dato, riprendere la cospicua letteratura critica intorno a Das Unheimliche nelle interrelazioni con la funzione poetica del linguaggio, che ci permettiamo di dare per acquisita quantomeno nelle linee portanti. 


\section{Durs Grünbein. Un'idea di Dante 3D \\ Rosalba Maletta}

denen Kunst und Philosophie sich seit langem abarbeiten. Mein einziges Mitspracherecht ist die Poesie. Auf sie muß ich mich verlassen können, und vice versa. Sie verlangt nach der Überraschung, sie sucht das geistige Abenteuer, die Verblüffung, darf alle ihr zur Verfügung stehenden Ausdruckstechniken anwenden. Die Poesie gestattet es einem, Sprünge zu machen, sich als Känguruh durch die Landschaften der Imagination zu bewegen. (Grünbein; Eskin 391)

Come lettore mi piace segnare passaggi che posso collegare con quelli di altri libri. Ciò crea una rete di loci testuali che si adattano, si completano e si ampliano reciprocamente. Ho sempre auspicato che anche per i miei scritti il lettore procedesse in tal modo. Mi vedo come parte di un continuum di idee rilevanti su cui arte e filosofia lavorano sodo da parecchio tempo. La mia sola voce in capitolo è la poesia. Debbo poter contare su di essa e viceversa. Essa esige la sorpresa, persegue l'avventura spirituale, lo stupore; le è dato utilizzare tutte le tecniche espressive a sua disposizione. La poesia permette di fare dei salti, di muoversi come un canguro attraverso i paesaggi dell'immaginazione.

Viene in mente ciò che scrive Rancière a proposito del sistema di evidenze atto a determinare una politica dei discorsi (12-14). La scrittura distrugge il rapporto tra gli effetti della parola e la posizione dei corpi nello spazio comune. La superficie di segni muti circola indiscriminatamente e può capitare in mano a qualsiasi corpo in qualsiasi posizione (15-17). Quanto captiamo mediante i sensi porta per contro a una suddivisione dei tempi e degli spazi, del visibile e dell'invisibile, della parola e del rumore come entità separate e distinte. Se ne deduce che la proscrizione della poesia dichiarata da Platone, prima di fondarsi sul contenuto immorale delle favole si fonda sull'impossibilità di controllare la parola scritta.

C'è un partito preso della poesia che comporta la negazione di qualsivoglia rapporto di necessità tra una forma e un contenuto predeterminati (17). Infrangendo la rigida suddivisione stabilita dal controllo sensoriale, ogni gerarchia rappresentazionale viene distrutta. La comunità dei lettori si istituisce in quanto sancita dalla mera circolazione della lettera.

Se intendiamo lettera nell'accezione di Lacan, come ciò che erode il discorso introducendo una dimensione incontrollabile e ignota, la circolazione aleatoria della lettera rappresenta il paradosso di una contingenza destinata a durare. ${ }^{9}$ Questo paradosso costituisce il nucleo più intimo e verace di ogni singola poesia:

Was ist es, das in der Dichtersprache alles bloß Körperliche überlebt? Wie spricht in ihr Psyche sich so aus, daß sie den lebendigen Körper abhebt vom bloßen Kadaver?

Das alles mag sehr weit hergeholt scheinen, tatsächlich aber liegt hier der Schlüssel zu einem magischen Zwischenreich, in dem auch die Worte des Dichters zum Leben erweckt werden. Denn im Gedicht geht es nicht um bloße Wörterbuchworte, sondern solche mit einer bestimmten psychischen Ladung. Die Vokabeln des Verses stehen gewissermaßen in einem elektromagnetischen Feld, ihre Operatoren könnten daher eben jene Lebensgeister sein oder etwas ihnen Entsprechendes, von dem uns vor Zeiten die Schamanen erzählten, erst gestern die Physiker — und heute, in ihrer Terminologie, die Hirnforscher.(Der cartesische Taucher 115-116)

Che cosa sopravvive nel linguaggio poetico a tutto quanto vi è di meramente fisico? Come si esprime la psiche perché sia possibile distinguere il corpo vivo dal semplice cadavere?

Tutto ciò può sembrare del tutto inverisimile, eppure qui si trova di fatto la chiave di un magico regno intermedio, in cui anche le parole del poeta prendono vita. Perché in una poesia non si tratta semplicemente dei vocaboli del dizionario bensì di parole con una certa carica psichica. I vocaboli del verso stanno, per così dire, in un campo elettromagnetico, i cui operatori po-

\footnotetext{
9 Jacques Lacan elabora l'istanza della lettera a partire da "L'instance de la lettre dans l'inconscient ou la raison d'après Freud” (1957) sino a Le Séminaire. Encore 1972-1973. Livre XX (31 e passim) come pure nel densissimo "L'étourdit" (1973).
} 


\title{
Durs Grünbein. Un'idea di Dante 3D \\ Rosalba Maletta
}

trebbero essere proprio quegli spiriti vitali o qualcosa di simile, di cui gli sciamani ci narravano molto tempo fa, solo ieri i fisici - e oggi, con la loro terminologia, gli studiosi del cervello.

Ecco perché una scrittura che metta in circolazione la funzione poetica del linguaggio, esige che il suo autore rimanga saldamente ancorato alla lingua materna. La posta in gioco è alta poiché la voce, il timbro, la sequenza fonico-acustica, irriducibili al silenzio, irradiano dal centro stesso della lingua come sistema di segni condiviso e, al tempo stesso, conteso dal poeta (Grünbein, "Sterne, Städte, Gehirne" 424).

Questa capacità visionaria, che i poeti si trasmettono, eccede le tecnologie più ultimative. Attraversandole le surclassa in una figurabilità del corpo-carne che porta alla luce i secreti sedimentati in parole di parole:

\begin{abstract}
Man stelle sich vor, es gäbe ein Denken, das an bestimmte, sonst nur schwer zugängliche Stellen kommt, wie Zahnseide zwischen die hinteren Backenzähne oder ein Endoskop in den Magen. Gewisse Stellen wird es überhaupt zum ersten mal anschaulich machen, einzelne Nebengänge des unüberschaubaren seelischen Höhlensystems, das sich durch die Körper aller Menschen zieht und nur durch findige, kühn in die noch ungesicherter Stollen vorstoßende Phantasie entdeckt werden kann. Dieses Denken ist das poetische Denken und es ist keine Domäne der Dichter und Literaten, vielmehr die Methode vieler kleiner Suchtrupps, die aus verschiedenen Richtungen aufgebrochen sind, ohne voneinander zu wissen, ein Heer von Phänomenologen, das daran arbeitet, die uns allen gemeinsame Vorstellungswelt zu erweitern. ("Das Gedicht und sein Geheimnis" 93 - c. m.)
\end{abstract}

Immaginiamo che ci sia un pensiero in grado di raggiungere determinate zone altrimenti impervie, come il filo interdentale tra i molari posteriori o un endoscopio nello stomaco. Esso renderà visibili per la prima volta certi punti, singoli passaggi laterali dello sconfinato sistema di caverne mentali che attraversa il corpo di tutti gli esseri umani e che può essere scoperto solo da un'immaginazione intraprendente che coraggiosamente si fa strada tra i cunicoli ancora incustoditi. Questo è il pensiero poetico; esso non è il dominio di poeti e letterati, piuttosto il metodo di tante piccole squadre di ricerca che si sono messe in cammino da diverse direzioni senza sapere l'una dell'altra, un esercito di fenomenologi al lavoro per ampliare il mondo immaginario comune a tutti noi.

La metafora, alquanto prosastica, memore della disciplina igienista della DDR, è tipica di un poeta che legge e rilegge Dante ma ha attraversato Beckett e guarda alla Cantica delle delizie celesti con Belacqua Shuah. ${ }^{10}$ Segue una definizione del pensiero creativo che risente della lezione di Descartes e di Husserl ma come non riconoscervi pure echi dell'Anabasi di Senofonte, magari riletta con Borchardt, se infine Grünbein ne parla come del metodo ( $\mu \varepsilon \tau \alpha-$ $o ́ \delta o ́ \varsigma)$ di molteplici piccole squadre di ricerca che, senza sapere l'una dell'altra, si mettono in viaggio da diverse direzioni con l'intento di ampliare il nostro mondo rappresentazionale?

Soffermiamoci sul composto nominale "Vorstellungswelt". In una prospettiva freudiana è il mondo legato alla rappresentazione di cosa, allorché questa aggancia la rappresentazione di parola per muovere verso l'illusione condivisa, in base alla quale nomina sunt consequentia rerum. ${ }^{11}$

10 Avanzo un parallelo tra la postura sapiente del Belacqua dantesco, Grünbein la sera della caduta del Muro di Berlino evidenziando il legame con Beckett in Maletta 2020. Ho sviluppato parallelo e argomentazioni in Maletta 2021. Nella conversazione-intervista con Ruzzenenti, Grünbein parla della voce di Dante in relazione a Dante And The Lobster (1934) di Beckett.

11 «e perché fosse qual era in costrutto, / quinci si mosse spirito a nomarlo / del possessivo di cui era tutto. // Domenico fu detto; e io ne parlo / sì come de l'agricola che Cristo / elesse a l'orto suo per aiutarlo" (Paradiso XII, vv. 67-72, p. 158 e nota 66). Nel Paradiso è San Domenico a riassumere in sé 


\section{Durs Grünbein. Un'idea di Dante 3D \\ Rosalba Maletta}

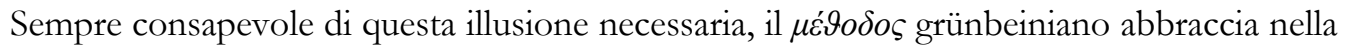
scia di Dante, aspetti dell'umano separati da un iperspecialismo disciplinare: psicologia, sociologia, teologia, economia, politica, estetica ("Das Punktum des Gedichts" 90-92). Una sventagliata di pratiche e saperi, dove non è possibile prescindere dall'interazione tra l'individuo e il suo tempo, in un itinerario in cui le coordinate spazio-temporali sono superate per seguire un disegno poetico che attinge a un passato immemoriale.

\section{Rendering}

Nella nostra epoca, immersa nella traduzione dell'analogico nel digitale, il percorso si definisce per via di rendering:

Hier setzt meine Übersetzung an, sie ist das, was man im Deutschen eine Übertragung nennt, im Englischen rendering, was in der Sprache der Architekten von heute eine Form der Visualisierung bedeutet, einen Entwurf in 3D. Darum geht es mir immer: eine bildliche Vergegenwärtigung. Regisseure, die das Leben Dantes verfilmen müßten, eine Szene aus seiner Jugend (mit oder ohne Kolorit und Kostüm) würden so vorgehen. Wie läßt sich das Geschehen so darstellen, daß ein Kinopublikum im einundzwanzigsten Jahrhundert es in aller Frische der ursprünglichen Situation wiedererkennen könnte? (Grünbein, "Ein flotter Sechser" - c. m.)

È qui che entra in gioco la mia traduzione, è quello che in tedesco chiamiamo una trasposizione, in inglese rendering che nel linguaggio degli architetti odierni designa una forma di visualizzazione, un disegno 3D. Per me si tratta sempre di ciò: una presentificazione per immagini. I registi che dovessero filmare la vita di Dante, una scena della sua giovinezza (con o senza colore e costumi) procederebbero in questo modo. Come presentare l'evento in maniera che un pubblico cinematografico del ventunesimo secolo possa riconoscerlo in tutta la freschezza della situazione originaria?

Così l'autore a proposito della resa in lingua tedesca del sonetto Guido, i' vorrei. ${ }^{12}$

Nella computer-grafica rendering identifica il processo di generazione dell'immagine a partire dalla descrizione matematica di una scena tridimensionale. Mediante il calcolo di tutti i punti prospettici, l'aggiunta di colori e ombreggiature, l'immagine bidimensionale risulta più realistica. Un rendering rafforza l'illusione della presenza dell'oggetto.

L'aspetto particolarmente interessante della terminologia qui impiegata da Grünbein risiede nel fatto che, a differenza della copia a mano o della riproduzione fotografica, il rendering illustra un modello che ancora non esiste come se esistesse. Viene in mente l'apologo di Zeusi e Parrasio, dove a ingannare gli uccelli è l'uva dipinta da Zeusi mentre a ingannare l'umano è il drappo dipinto da Parrasio.

Il significante rendering crea in questo senso un interessante cortocircuito con la locuzione die unheimlichste Stelle, con cui Grünbein introduce e compendia la terzina posta al culmine del viaggio ultraterreno di Dante, locus che lo impegna da un certo numero di anni:

Un punto solo m’è maggior letargo

Che venticinque secoli a la 'mpresa,

Che fé Nettuno ammirar l'ombra d'Argo. (Paradiso XXXIII, vv. 94-96)

quel che Dante scrive in Vita nuova: «che li nomi seguitino le nominate cose, sì come è scritto: Nomina sunt consequentia rerum» (Vita nuova XIII 20; XXIV 46).

12 Il sonetto, celeberrimo, della cui traduzione da parte di Grünbein non possiamo occuparci nel contesto dato, è in Rime, 9 (LII) 35-36. 


\section{Durs Grünbein. Un'idea di Dante 3D \\ Rosalba Maletta}

Riporto di seguito le due trasposizioni. Quella del 2021:

Ein wenig Schlafsucht bringt mich mehr ins Arge

als zweieinhalb Jahrtausende zuvor der Schatten,

der Neptun staunen ließ: die Heldenfahrt der Argo. ("Die unheimlichste Stelle")

Quella del 2009 — inserita nella nona "immersione" di Die Bars von Atlantis, significativamente intitolata "Meerfahrt mit Dante":13

Ein Punkt kann mehr Entrücktheit mir bereiten

Als zweieinhalb Jahrtausend bei dem Zug,

Da starr Neptun sah Argo's Schatten gleiten. (42)

Assai significativo per la nostra analisi è che un rendering sia predisposto per catturare l'immagine in base al modo in cui la luce colpisce gli oggetti. ${ }^{14}$ La tecnica impiegata non si limita infatti a considerare l'illuminazione diretta. Elabora un algoritmo, noto come illuminazione globale ovvero indiretta, atto a processare tutte le fonti di luce,anche quelle di cui non siamo consapevoli e che i nostri sensi non sono in grado di percepire. ${ }^{15}$

Il passaggio di testimone tra Dante e Grünbein è produttivamente rilanciato. La proiezione $3 \mathrm{D}$, che il poeta mette a punto nella sua mente per accendere la nostra immaginazione, è volta a restituire quel $\kappa o ́ \sigma \mu o \varsigma$, dove la parola esplode in ogni direzione per esprimere il percetto nel momento stesso della Wahrnebmung. Questa a sua volta si presenta tanto in Grünbein quanto in Dante affatto scevra di idealizzazioni come pure di nostalgie retrospettive, dunque profondamente calata nella propria epoca.

\section{Dante psichedelico}

In un saggio del 1995 Grünbein tratteggia «un'idea di Dante» in linea con la rivoluzione introdotta in ambito percettivo, psichico e cognitivo dalla meccanica quantistica e dalla fisica delle particelle:

[...] er ist der Kartograph eines Weltinnenraums, sein Weltbild ist weder geozentrisch noch kopernikanisch, sondern in einem sehr frühen, sehr wirksamen Sinn: psychedelisch. Man erkennt es an den Echos, die von allen Seiten herandrängen, an der magnetischen Methode, mit der hier Raumklänge und Wegzeichen, Terrestrisches und Zerebrales, Halluzination und Konstruktion parataktisch übereinander herfallen. ("Galilei vermißt Dantes Hölle und bleibt an den Maaßen hängen" 97 - c. m.)

[...] è il cartografo di uno spazio interiore del mondo, la sua immagine del mondo non è né geocentrica né copernicana bensì, in un senso assai precoce, molto incisivo: psichedelica. Lo si ri-

13 Per "Meerfahrt mit Dante" cfr. Maletta 2021293 ss.

${ }^{14} \mathrm{Si}$ veda la definizione in Garzanti, in particolare al punto 5: «(inform.) rendering $(\mathrm{m}$.) (generazione di un'immagine grafica sulla base di un modello matematico di un oggetto o di una scena tridimensionale). - (inform.) procedura che permette di generare, con un apposito programma, un'immagine digitale a partire da una serie di informazioni (descrizione degli oggetti tridimensionali, punto di vista, illuminazione ecc.); è molto usata per creare video digitali, videogiochi, effetti speciali per cinema e televisione, e in ingegneria e architettura per creare modelli digitali di edifici o aree urbanizzate $\mid$ immagine prodotta con questa procedura»: https://www.garzantilinguistica.it/ricerca/?q=rendering

15 Cfr. il sito MKRLAB: https://www.mkrlab.eu/2020/02/06/che-cose-il-rendering-3d/ (ultima consultazione 28.09.2021). 


\section{Durs Grünbein. Un'idea di Dante 3D \\ Rosalba Maletta}

conosce dagli echi che si ammassano da ogni dove, dal magnetismo, con cui i suoni dello spazio e i segnavia, quanto proviene dalla terra e dal cervello, allucinazione e costruzione paratatticamente si avventano l'uno sull'altro.

Se il composto Weltinnenraum è hapax rilkiano ${ }^{16} \mathrm{e}$ va pensato insieme a quel che Grünbein chiama, con Cummings, enormous room ("Reflex und Exegese" 64), l'immagine del mondo che Dante consegna ai posteri non è geocentrica ovvero copernicana bensì - in un'accezione primeva e attiva (wirksam nel senso dello čpyov aristotelico) - psichedelica ("Galilei ver-

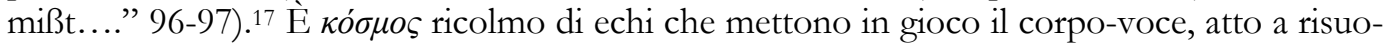
nare di sensazioni, emozioni, percetti, Affekte che urgono per essere legati (nel senso della Bändigung e del lavoro di eros freudiani).

Il corpus della Commedia è interamente attraversato dal linguaggio, sussiste nel significante proprio perché le immagini non lo saturano, i sensi non lo ingannano. Anche in tale accezione è psichedelico. Prossimo alla fisica delle particelle e all'ipersfera, ${ }^{18}$ schiude spazi immaginativi all'invisibile e all'antimateria.

Per Grünbein Dante è il tuffatore delfico, forse proprio così come Socrate se l'è figurato nella ricerca dei tesori che gli scritti di Eraclito trattengono, ma è pure Ionà nel ventre della balena, un secondo Teseo nel labirinto e ancora, in maniera affatto moderna, un'incarnazione di tutti i viandanti degli inferi e scalatori di monti da Odisseo a Empedocle: «Immerfort unterwegs, ins Gespräch vertieft oder neugierig den Gefolterten zugewandt, ist Dante der lebende Beweis für den Prozeßcharakter aller wirklichen Künste, für ihr kinetisches, taktiles, physiologisches Arbeiten» - «Perennemente in cammino, immerso nel dialogo o rivolto con curiosità ai suppliziati, Dante è la dimostrazione vivente del carattere processuale di tutte le vere arti, del loro lavorio cinetico, tattile, fisiologico» (“Galilei vermißt..." 97).

Nessuna misurazione neurofisiologica potrà mai spiegare i mondi che Grünbein incontra nel corso delle sue immersioni subacque e subconsce. Come nessuna combinazione neurochimica verrà a capo del viaggio di Dante; piuttosto un'idea di "traduzione" come poeticamente la mette in opera lo scrittore tedesco, figlio di un esilio vissuto all'ombra del Muro di Berlino.

Mandel'štàm - ci fa notare Grünbein - celebra in Dante il maestro inarrivato del confronto e delle analogie e con una di quelle anacronie anasemiche che sono il sale della poesia egli lo definisce «il Descartes della metafora» ("Galilei vermißt ..." 103). Ecco allora che per via commutativa Grünbein definisce Dante «il Descartes della teoria della conoscenza» (22): la Commedia ci permette di risalire al cominciamento della fenomenologia di un Io pensante che è pure un Io poetante.

Mediante il ricorso ai paragoni, alle metafore, a innumerevoli figure retoriche la poesia non riproduce la natura bensì la crea ogni volta di nuovo. E con ciò giungiamo a uno snodo decisivo nell'interpretazione e nella resa che Grünbein ci consegna della terzina in oggetto (Paradiso XXXIII, vv. 94-96).

Siamo nell'ultimo canto dell'ultima cantica. Di lì a poco la voce del poeta, dunque la sua scrittura si spegneranno nella contemplazione che travalica i limiti del dicibile, di quanto all'umano è dato racchiudere nelle parole, fossero pure quelle della poesia, della preghiera, della devozione. La materia per Mandel'štàm si rivela nel procedere per confronti (Vergleiche)

16 Rilke, “Es winkt zu Fühlung”, composta nel 1914, pubblicata postuma (Werke II 113).

${ }^{17}$ Sul lessema "psichedelico" e sue derivazioni cfr. Maletta 2021290 ss.

18 Dagli occhi di Beatrice all'ipersfera di Riemann alla cosmologia quantistica si muovono le considerazioni di Patapievici, come già rilevavo nel saggio di cui sopra. 


\title{
Durs Grünbein. Un'idea di Dante 3D \\ Rosalba Maletta
}

e la dimensione dell'Essere consiste di questa floridezza e fluidità delle immagini (Bildlichkeit) (Der cartesische Taucher 21). ${ }^{19}$

Il bello dei paragoni, rileva Grünbein, è che in ogni momento è possibile invertirne le polarità. Prestiamo attenzione a questa figura, le cui polarità risultano intercommutabili:

\begin{abstract}
Mandelstams Spruch, um 180 Grad gedreht, könnte also lauten: Wenn Dante der Descartes der Metapher war, dann war Descartes - der Dante der Erkenntnisheorie. Ein Philosoph, der wie jeder gute Dichter sein Werk mit Selbstbeobachtung anfing. Bei ihm sagt das Denken, jenseits von Platon und Augustinus, zum ersten mal wieder ich. (Der cartesische Taucher 21-22) ${ }^{20}$
\end{abstract}

Il detto di Mandel'štàm, ruotato di 180 gradi, potrebbe quindi essere: se Dante fu il Descartes della metafora, allora Descartes fu il Dante della teoria della conoscenza. Un filosofo che, come ogni valente poeta, comincia la sua opera dall'osservazione di sé. Con lui, al di là di Platone e di Agostino, il pensiero dice per la prima volta di nuovo "Io".

Appena prima Dante viene salutato come colui che ha reso possibile emanciparsi da un immaginario irrigidito e catalogico, per così dire prefabbricato. Con lui le immagini imparano a correre, le parole assumono sembianti e colorature uniche, icasticamente connotate e le metafore - parole di parole che trasportano sensazioni, emozioni - fungono da variabili:

Püntlich ist mit ihm die antike Konfektion fertiger Abziehbilder, der Trick mit dem schmückenden Beiwort, beendet. Oder anders gesagt: es ist Dante, bei dem die Bilder laufen lernen und Metaphern zu Variablen werden innerhalb einer poetischen Ontologie.(21)

In maniera puntuale con lui si conclude l'antica moda delle immagini già pronte, l'espediente dell'epiteto esornativo. O detto altrimenti: è con Dante che le immagini imparano a camminare e le metafore divengono variabili all'interno di un'ontologia poetica.

Prestiamo attenzione all'avverbio derivato pünktlich che esprime la capacità di non mancare l'appuntamento con il novum ma, nella formula antiquata che sta per "gewissenhaft; korrekt", ${ }^{21}$ veicola pure il modo con cui viene introdotto questo novum, che in fondo lo stesso Grünbein si ascrive rinvenendolo nell'estetica di Vischer. Qui egli rinviene: «eine physikalisch-technische Erklärung der Poesie, avant la lettre. Die lyrische Dichtung sei "punctuelles Zünden im Subjecte"» - «una spiegazione fisico-tecnica della poesia, avant la lettre. La poesia lirica sarebbe "accensione puntuale nel soggetto"» ("Das Punktum des Gedichts" 96 - c. m.).

È un'accensione repentina e subitanea, come in Zündkerzen, la raccolta poetica del 2017, dove Grünbein inanella istantanee, "candele di accensione" mediante cui il mondo "viene all'idea" per un singolo soggetto in quella prima e unica volta: «Letzte Chance und einzige, etwas zu sagen: das ist das Gedicht. Anscheinend bin ich der mystische Mensch, der an den Augenblick glaubt, da etwas sich mitteilt, etwas mitteilenswert ist» - «Ultima occasione, la sola per dire qualcosa: questo è la poesia. A quanto pare sono l'uomo mistico che crede nell'istante in cui qualcosa si comunica, qualcosa diventa degno di essere trasmesso ad altri» (97).

${ }_{19}$ Osip Mandel'štàm pone il pensiero dei grandi naturalisti à la Darwin in connessione con Dante che definisce, in quanto loro predecessore, il «Descartes della metafora». L'immagine è ripresa da Grünbein nei materiali preparatori a Vom Schnee oder Descartes inDeutschland (2003), in particolare nella Vorrede (p. 543), e analizzata in Eskin (2007). Una rivisitazione della stessa, è in Der cartesische Taucher (21).

${ }^{20}$ Con minime variazioni nella "Vorrede" a Vom Schneeoder Descartes in Deutschland 543-544

21 "Pünktlich": DUDEN: https://www.duden.de/rechtschreibung/puenktlich (ultima consultazione 28.09.2021); GRIMM: Deutsches Wörterbuch von Jacob Grimm und Wilhelm Grimm ( $\left.{ }^{1} \mathrm{DWB}\right)$ : https://www.dwds.de/wb/dwb/p\%C3\%BCnktlich (ultima consultazione 28.09.2021). 


\section{Durs Grünbein. Un'idea di Dante 3D \\ Rosalba Maletta}

\section{Idioma materno 3D}

Omai sarà più corta mia favella pur a quel ch'io ricordo, che d'un fante, che bagni ancor la lingua a la mammella. (Paradiso XXXIII, vv. 106-108)

Was gemeint ist heißt Name, was verschwiegen bleibt Ding. Weitverzweigt sind die Sätze zu jeder Schandtat bereit. ${ }^{22}$

Nel commento che nel 2021 dedica alla sua trasposizione in lingua tedesca di Paradiso XXXIII, vv. 94-96 Grünbein sceglie di allontanarsi dalla via percorsa nel 2009. In Meerfabt mit Dante la corrispondenza con la terzina dantesca appare maggiormente aderente e puntuale sul piano letterale come pure su quello della scelta di un lessico più aulico e ricercato:

Es ist der Moment, da Mythos und Geschichte in einer Art Wachablösung sich kreuzen, ein absoluter Knotenpunkt, den wie immer der Reim markiert. [...] Staunend registriert der Gott, wie die Zukunft ihn einholt und überrollt.

Ein Punkt kann mehr Entrücktheit mir bereiten

Als zweieinhalb Jahrtausend bei dem Zug,

Da starr Neptun sah Argo's Schatten gleiten.

È il momento in cui mito e storia si intersecano in una specie di cambio della guardia. Un punto cruciale assoluto, che come sempre è messo in rilievo dalla rima. [...] Sbalordito, il dio percepisce come il futuro lo raggiunga e lo travolga.

Un punto solo m’è maggior letargo

Che venticinque secoli a la 'mpresa,

che fé Nettuno ammirar l'ombra d'Argo. ("Meerfahrt mit Dante” 42 - c. m.)

C'è da chiedersi chi guardi e da quale vertice prospettico. Ipotizziamo una verticalità che interseca un'orizzontalità che comporta pure una rifrazione. L'oggetto-nave produce ombra in quanto corpo solido.

Un'imbarcazione è pure in Guido, i' vorrei. Un "vasel" che Grünbein attualizza in "Segler" dalle molteplici connotazioni, tra cui pure quella di colui che conduce la barca a vela. ${ }^{23}$ L'«umanista misantropo» ${ }^{24}$ rivisita l'invito che Dante, giovane spensierato ben lungi dall'idea dell'esilio, rivolge agli amici più cari. Il sonetto Guido, i' vorrei esprime nell'idioma materno i desideri più intimi e riposti. Essi fanno capolino nel volgare di Dante che, svincolato dai modelli precedenti, vibra nelle Rime:

Eine neue, unerbörte Musikalität der Verssprache ist das Markenzeichen. Das ging über das allgemeine Schullatein hinaus, für den natürlichen Klang brauchte es das Italienische, die Mutter-

22 «Quanto è significato si chiama nome, quanto / è sottaciuto rimane cosa. / Ampiamente ramificate le frasi sono / pronte a ogni oltraggio" (Grünbein, "Fisch im Medium").

23 DUDEN ONLINE: https://www.duden.de/rechtschreibung/Segler (ultima consultazione 28.09.2021).

${ }^{24}$ Misanthropischer Humanist è il titolo della lirica che chiude la sezione 6 di Zündkerzen (120). 


\section{Durs Grünbein. Un'idea di Dante 3D \\ Rosalba Maletta}

sprache, den Jargon, den alle Volksschichten benutzten und den man überall auf den Straßen zu hören bekam. Eine Sprache, in der sich die geheimsten Wünsche zum ersten Mal aussprechen ließen: "Guido, i'vorrei ...”. (“Ein flotter Sechser" - c. m.)

Il segno distintivo è una nuova, inaudita musicalità del linguaggio in versi. Ciò andava oltre il comune latino scolastico, per il suono naturale ci voleva l'italiano, la lingua materna, il gergo usato da tutti i ceti popolari e che si udiva ovunque in strada. Una lingua in cui i desideri più riposti potessero venir espressi per la prima volta: "Guido, i' vorrei ...".

Lungo questa riflessione si inserisce il rendering del poeta che deve traghettare l'idioma di Dante in un'altra lingua, la sua propria lingua materna, di ceppo differente rispetto alle lingue romanze, e nella sua propria epoca che è la nostra di Europei in piena rivoluzione digitale.

Se ne ricava una proiezione in 3D, poiché si tratta di adeguare la situazione alla contemporaneità. In questo contesto Grünbein fornisce pure una chiave di lettura per la nostra terzina: «Reime formstreng zu übertragen ist für den Übersetzter eine Folter - ich meine, es genügt, sie anzudeuten, sie gleichsam anzukanten, damit das Boot wirklich zum Schaukeln kommt» (Ibidem)

Accennare le rime della lingua dell'Altro ricorrendo alla propria comporta un lavoro di sponda e bordo: il poeta ricevente si mette a spigolo e canto per far sì che la barca, l'arca delle parole, cominci a oscillare altalenando moto e ritmo. Come non pensare al Fort/Da di Freud in cui l'umano nasce al linguaggio e si lascia abitare da quei significanti ritagliati in univocità di rinunzia, che il gioco poetico rimette ogni volta in discussione?25

\section{Il punto ideale}

Torniamo alla nostra terzina: questi mezzi consentono di instaurare una corrispondenza tra oggetto (o entità) e descrizione di posizione, ma anche tra descrizione e oggetto in un rapporto di corrispondenza biunivoca che nel 2009 Grünbein rende in maniera più puntuale e astratta:

Ein Punkt kann mehr Entrücktheit mir bereiten

Als zweieinhalb Jahrtausend bei dem Zug,

Da starr Neptun sah Argo's Schatten gleiten.

Un punto solo m’è maggior letargo

Che venticinque secoli a la 'mpresa,

che fé Nettuno ammirar l'ombra d'Argo.

Il punto compare in traduzione speculare: una figura non ulteriormente scomponibile e priva di dimensioni. Un punto che riceve la possibilità di esistere secondo le coordinate proiettive cartesiane e, nell'anacronia volutamente perseguita da Grünbein, un punto che è nello spaziotempo mobilissimo.

${ }^{25}$ Nel VII capitolo della Traumdentung Freud scrive: «Ich stelle mir vor, daß der bewußte Wunsch nur dann zum Traumerreger wird, wenn es ihm gelingt, einen gleichlautenden unbewußten zu wecken, durch den er sich verstärkt» (558) - «Mi figuro che il desiderio consciente stimoli il sogno, solo se riesce a risvegliare un desiderio inconscio consonante, mediante il quale si rafforza». Il participio presente in funzione attributiva "gleichlautend" ci invita a porre l'accento sulla dimensione acustica, prettamente sonora dell'evento, atta a risvegliare ritmo e armonie immemori. Per il gioco del Fort / Da il riferimento è a "Jenseits des Lustprinzips" (1920, 11-15). 


\section{Durs Grünbein. Un'idea di Dante 3D \\ Rosalba Maletta}

Abbiamo già scritto che Grünbein definisce Descartes «der Dante der Erkenntnisheorie» (Der cartesische Taucher 21-22) poiché i suoi studi permettono alla mente e all'Io di traghettare i pensieri verso astrazioni mai prima osate. Gettando le fondamenta della geometria analitica e del dubbio, in cui il soggetto si riconosce come tale, Descartes amplia gli spazi dell'immaginazione con procedimento rigoroso e, al tempo stesso, spettrale.

Ai solidi di Galileo egli sostituisce le lettere dell'alfabeto, diagrammi, grafici, vettori e funzioni. Mostra e dimostra il processo del pensiero mentre accade, così come Dante ci presenta il processo della inventio mentre si va svolgendo:

Nur wenige - elegante Fechter wie Dante - haben das Gleichnis als image juste immer genau dort gesehen, wo es wie von selbst aus der Dynamik des Geschehens hervorstach, nur wenige haben den Gedanken im Entstehen erfaßt und festgehalten, das Bild im Moment seines natürlichen Auftauchens bewahrt.("Mein babylonisches Hirn” 28)

Solo pochi - eleganti schermidori come Dante - hanno sempre visto la similitudine quale image juste proprio là, dove essa risaltava come da sé dalla dinamica dell'accadimento. Solamente pochi hanno colto e fermato il pensiero in divenire, serbato l'immagine nel momento della sua naturale emersione in superficie.

Un punto determina pertanto non solo le coordinate e le proiezioni degli assi cartesiani ma statuisce pure un tempo immaginario, come nelle odierne speculazioni della cosmologia quantistica. ${ }^{26}$ Un punto in cui il soggetto osservante modifica l'oggetto osservato modificando se stesso. Intorno a questo punto si dispone la forma della poesia:

Das Gedicht wird nicht von selbst belebt in der zersprengten Odenform, es erstarrt nicht, nur weil es sich in Reihen formiert. Entscheidend ist der Weg von Punkt A der Imagination zu Punkt B, und wenn Form grausamer als Vertrauen ist, dann ist die Dimension wandelbarer als Form. Wie in der Physik ist die Dimension einer Größe etwas, das sich erst durch ein Überspringen ergibt, durch das Wagnis oder die Willkür eines Gleichheitszeichens. In der so einfachen wie folgenreichen Formel Geschwindigkeit = Weg : Zeit ist es das Gleichheitszeichen, das den Verkehr zwischen den Dimensionen regelt. ("Brief über den Sarkasmus und das Gedicht als Konzept” 51-52)

La poesia non si anima da sola nella forma spezzata dell'ode, non si irrigidisce solo perché si modella in righe. Decisivo è il percorso dal punto $\mathrm{A}$ dell'immaginazione al punto $\mathrm{B}$, e se la forma è più crudele della fiducia, allora la dimensione è più mutevole della forma. Come nella fisica, la dimensione di una grandezza è qualcosa che si ottiene solo con un salto, per l'azzardo o l'arbitrarietà di un segno di uguaglianza. Nella formula, tanto semplice quanto densa di conseguenze, $\mathrm{v}=\mathrm{s} \div \mathrm{t}$, è il segno di uguaglianza a regolare il rapporto tra le dimensioni.

E possiamo continuare sempre con le parole di Grünbein, il quale in Mein babylonisches Hirn circoscrive questo spazio puntuale:

Denn das Wort ist physischen Ursprungs. Nur das innegewordene Wort schützt vor der erinnerungslosen Alltagssprache, es hält die Verbindung zum Einmaligen, zur Idiographie primärer Wahrnehmung. Im Gedicht drängt zuallererst sich das Schreckliche eines eingeschlossenen Subjekts auf, das mit ganzer Überredungskunst nach außen zu dringen sucht. Seine Schönheit beginnt als Verwirrung, Unzulänglichkeit, Engstirnigkeit, vielleicht böse, fast immer nervös sich äußernde Sinlichkeit und endet als ein Maximum an Persönlichkeit, Esprit, Affekt, Vorlieben und Eigensinn, - auf kleinstmöglichem Raum. (18-19 - c. m.)

${ }^{26}$ Si vedano gli studi di Penrose. 


\section{Durs Grünbein. Un'idea di Dante 3D \\ Rosalba Maletta}

Perché l'origine della parola è fisica. Solo la parola interiorizzata ci protegge dal linguaggio senza memoria della vita quotidiana; mantiene il legame con l'unicità, con l'idiografia della percezione primaria. Nella poesia si impone prima di tutto l'orrore di un soggetto segregato che cerca di penetrare all'esterno con tutta l'arte della persuasione. La sua bellezza comincia come confusione, inadeguatezza, ristrettezza mentale - con una sensualità espressa forse con perfidia, quasi sempre in maniera nervosa - e finisce con un massimo di personalità, esprit, Affekt, preferenze e ostinazione - nel minor spazio possibile.

\section{Indagine quantitativa vs dissonanze fisiologiche}

In Galilei vermißt Dantes' Hölle und bleibt an den Maaßen hängen Grünbein istituisce un confronto interessante tra le verità della Nova Scientiae il viaggio nella meta-physis e nella mistica dei numeri del cosmo dantesco (101).

Nato lo stesso anno di Shakespeare e tre giorni prima di Michelangelo (92), Galileo introduce il concetto di spazio e di quantificazione nella poesia dell'Inferno, certo di esibire una localizzazione precisa assieme alla misura esatta delle ali di Lucifero (96).

Espunti l'eccedenza, i pathemata, la mitologia, le ridondanze teologiche dalle ferree leggi del calcolo, non rimangono che imbuti, sfere, cilindri (95): «Physikalische Konsonanz schlägt um in physiologische Dissonanz» - «La consonanza della fisica si capovolge in dissonanza fisiologica» (100).

Quel che distingue, nella maniera più netta e marcata Galileo da Dante è la domanda che Grünbein pone a se stesso e a noi lettori: «Woher dieses Verlangen nach einem rücksichtslosen a priori, das ihn auf alle Vielfalt und jede Besonderheit herabsehen ließ, mit den Glasaugen der physikalischen Vorsehung?» - «Donde questo anelito verso uno spietato a priori che, attraverso gli occhi di vetro della provvidenza della fisica, gli faceva guardare con degnazione a ogni manifestazione del molteplice e del particolare?» (93).

La provvidenza della nuova scienza - con il suo computo esatto, la conquista dello spazio oggettivato e oggettivante - confligge con la poetica fisiologica dell'universo dantesco:

Mit der Entdeckung der Pendelgesetze wird der menschliche Körper synchronisiert; die Medinizer erhalten ein Instrument zur Messung der Pulsrate. Längst gibt das Experiment, den Modellversuch: aus einem Körper wird eine Kugel, aus der Kugel der ideale Punkt. Von jedem Fallbeispiel bleibt schließlich nur mehr der Fall übrig: die Nuß kann taub sein, allein ihr Gehäuse ist wichtig. (93- c. m.)

Con la scoperta delle leggi del pendolo, il corpo umano viene sincronizzato; i medici ricevono uno strumento per misurare la frequenza cardiaca. Da tempo esiste l'esperimento, la prova del modello: un corpo si trasforma in una sfera, la sfera diventa il punto ideale. Alla fine, di ogni caso esemplificativo non rimane che il caso: la noce può essere vuota, ciò che conta è l'involucro.

Quanto lontano questo «punto ideale» da quello cantato da Dante e presente nella prima trasposizione di Grünbein, il quale risalendo alle proprie origini non trascura di menzionare, insieme a Borges e a Curtius, Filalete ossia "il nestore della dantistica in Germania" (Waentig 315).

Nello scritto del 2009, in cui compare la prima versione di Paradiso XXXIII, vv. 94-96, Grünbein presta particolare attenzione ai calcoli eseguiti dal sovrano sassone sui secoli trascorsi dall'impresa degli Argonauti al momento in cui Dante è quasi al culmine del suo viaggio.

Giovanni il Sassone, che aveva intrapreso studi giuridici, fu detto Philaletes, colui che ama la verità, ovvero Der Wahrhaftige per via del profondo senso della giustizia che esercitò sui sudditi anche grazie alla passione per Dante (Waentig $315 \mathrm{ss}$ ). La sua traduzione della Divina 


\section{Durs Grünbein. Un'idea di Dante 3D \\ Rosalba Maletta}

Commedia - per la quale consultò Carl Gustav Carus rispetto alle branche di medicina, scienza e anatomia, e Wilhelm Gotthelf Lohrmann per quelle dell'astronomia (Ivi) - costituisce a tutt'oggi un riferimento pregiato. Grünbein menziona l'edizione del 1865, anno del sesto centenario della nascita del poeta, in cui a Dresda viene fondata la Società dantesca. ${ }^{27}$

Nel richiamo alla figura del monarca illuminato della dinastia cattolica dei Wettin, le cui spoglie riposano nella cripta della Hofkirche di Dresda, ${ }^{28}$ Grünbein non manca di centrare un parallelo con il poeta degli scritti teorici e propedeutici alla Divina Commedia. Il De monarcbia, il Convivio, l'Epistola a Can Grande della Scala, la dibattuta Quaestio de aqua et terra (1320) danno prova di quella verità poetica che è sovente assai più inverisimile di qualsivoglia realtà pratico-operativa misurabile e quantificabile. ${ }^{29}$ Soprattutto statuisce una dimensione temporale in cui successioni e stirpi si avvicendano mostrando come la storia dell'uomo sia la storia delle conquiste dello spazio.

\section{Dal pathos allo Unheimliches}

Nel 2021 Grünbein giunge al punto in maniera differente:

$$
\begin{aligned}
& \text { Ein wenig Schlafsucht bringt mich mehr ins Arge } \\
& \text { als zweieinhalb Jahrtausende zuvor der Schatten, } \\
& \text { der Neptun staunen ließ: die Heldenfahrt der Argo. } \\
& \text { Un punto solo m'è maggior letargo } \\
& \text { che venticinque secoli a la 'mpresa } \\
& \text { che fé Nettuno ammirar l'ombra d'Argo. }
\end{aligned}
$$

Impossibile, nel confronto con il testo del 2009, sottacere il nome del grande romanista Ernst Robert Curtius, il quale nella terzina in questione individua - e Grünbein lo cita espressamente - «una situazione unica / eine einmalige Situation» nel corpus del sommo fiorentino. Curtius la definisce, al superlativo assoluto, «die pathetischste» ("Meerfahrt mit Dante" 42).

Dal pathos della «situazione» di Curtius a «die unheimlichste Stelle» c'è da chiedersi di quale transito e passaggio intenda riferirci Grünbein con il ricorso a un significante che, grazie a Freud, ha assunto valenza, colore e corredo di sfumature intraducibili, profondamente intrecciate con la natura più intima della creazione poetica e letteraria.

\footnotetext{
${ }^{27}$ Sulle edizioni si veda: Julius Petzholdt. Nel 1865 Johann Heinrich Friedrich Karl Witte insieme agli italianisti Karl Bähr di Dresda, Adolph Mussafia di Vienna e Franz Xaver Wegele di Würzburg fondò a Dresda la Deutsche Dante-Gesellschaft, che si costituì con il patrocinio di Giovanni di Sassonia il 14 settembre, giorno della morte del sommo poeta (Ibidem 327-328). Trattasi della più antica fra tutte le associazioni dantesche internazionali, di cui Witte - matematico, giureconsulto, traduttore e filosofo divenne il primo presidente. La sua traduzione della Divina Commedia, pubblicata nel 1865, rimane al pari di quella del Filalete opera di riferimento.

${ }_{28}$ Su Filalete, figlio di Carolina di Parma, la sua passione per Dante, la terra e l'idioma italiano cfr. Adolf-Altenberg.

29 Sulla verisimiglianza in Dante cfr. Barolini (2003, 11-35 e passim; 2012, specialmente 245-265). Richiamandosi a Singleton e Auerbach la studiosa statunitense traccia una proposta decisamente originale e ben documentata. In un'intervista rilasciata a Giorgio Manacorda, germanista, poeta e pubblicista, Attilio Bertolucci teneva a precisare come La camera da letto non fosse un testo autobiografico e aggiungeva: «Potrei dire che è inventato dal vero secondo una mappa disegnata in sogno».
} 


\section{Durs Grünbein. Un'idea di Dante 3D \\ Rosalba Maletta}

Il traghettamento linguistico dalla lingua materna di Dante a quella di Grünbein, dodici anni dopo aver ricordato la definizione di Curtius, implica la rilettura della terzina dantesca sullo sfondo di una nuova era, in cui vecchi idoli crollano e nuovi si ergono nell'orizzonte di una revolutio dove vero e verisimile si specchiano nel virtuale. La mutazione antropologica e antropocenica in atto chiama in causa lo Unheimliches come ciò che di più intimo e ignoto investe il processo di ominizzazione, l'umano in quanto soggetto di parola poiché soggetto alla parola. ${ }^{30}$

Un linguista del calibro di Rastier ascrive al linguaggio la potenza del distale che si estrinseca appieno nel finzionale, atto a descrivere domini inaccessibili all'esperienza immediata. Narrandoli, mettendoli in rima lo scrittore li istituisce, li chiama all'esistenza (14).

All'epoca delle proiezioni 3D e della realtà aumentata, la poesia mantiene per Grünbein questa funzione. Non cessa di istituirla e, salvaguardandola, di tramandarla. Si pensi al mero processo fisico descritto dal viator, pervenuto ai limiti dell'umana capacità di percepire mediante la vista:

\footnotetext{
Wieder zeigt es sich: Dante war einer der visuell wirkmächtigsten Dichter aller Zeiten. Wir sind im Schlußgesang. Gleich hat die Wanderung durch die drei Reiche ein Ende mit einem letzten Ausblick, der nur ihm als gewöhnlichem Erdenbürger mit eigenen Augen vergönnt war. Bevor der Erzähler, geblendet vom Licht, in stummer Himmelsschau zur Ruhe kommt, wirft er einen letzten Blick zurück - in die Tiefen des Ozeans. ("Die unheimlichste Stelle" - c. m.)

Di nuovo risulta evidente: Dante fu uno dei poeti visivamente più potenti di tutti i tempi.

Siamo nel Canto conclusivo. Tra un momento, il viaggio attraverso i tre regni si concluderà con un ultimo sguardo che solo a lui, cittadino comune della terra, è stato concesso esercitare con i propri occhi. Prima che il narratore, accecato dalla luce, si quieti nella muta contemplazione del cielo, eccolo lanciare indietro un'ultima occhiata - negli abissi dell'oceano.
}

L'occorrenza dei sostantivi e dei verbi afferenti al campo semantico della vista è sorprendente. Viene in mente quanto scrive Contini: «Il problema espressivo di Dante non è affatto quello di rappresentare uno spettacolo, bensì di enunciare, quasi teoreticamente, un'incarnazione di cose celesti e di descrivere l'effetto necessario sullo spettatore. A Dante qui non interessa punto un visibile, ma, ch'è tutt'altra cosa, una visibilità» ("Esercizio di interpretazione" 28-29).

\section{Per mare tra conquiste e passato immemoriale}

Al culmine del viaggio, giunto nell'Empireo, l'agens/auctor misura il cammino percorso. Si delinea una mise en abîme con il celebre passo del Convivio: «Veramente io sono stato legno sanza vela e sanza governo, portato a diversi porti e foci e liti dal vento secco che vapora la dolorosa povertade» (Convivio, I, III, 5, p. 50).

Grünbein, che ha esperito tempi difficili all'ombra del Muro, ${ }^{31}$ assurto a fama internazionale torna nel 2021 a osservare il pellegrino che, giunto al termine del viaggio, con un'ultima torsione, cerca le parole per descrivere quell'innalzarsi verso una dimensione altra, dove la forza di gravità non ha corso:

\footnotetext{
${ }^{30}$ Sullo Unheimliches in riferimento al multiverso digitale e digitalizzato cfr. Pascarelli.

31 Cfr. specialmente la raccolta Grauzone morgens; le considerazioni di "Kosmopolit" nonché Die Jahre im Zoo.
} 


\section{Durs Grünbein. Un'idea di Dante 3D \\ Rosalba Maletta}

Und nachdem alle Hürden genommen sind, kommt die Schwierigkeit, ohne weitere Hilfsmittel in den Himmel vorzustoßen. Hier extrapoliert Dante ein wenig, suspendiert das Problem der Schwerkraft. Er wird, in der Logik des Glaubens, einfach erhoben. Nachdem er die letzten Sphären - heute würde man sagen, die Stratosphäre, die Ionossphäre - durcheilt hat, kommt ein letzter Perspektivwechsel. Einer, der Raum und Zeit, die christliche und die grieschischmythische und selbst die historische [...] nochmals dreht in der Gegenüberstellung von Terra und Kosmos. ("Die unheimlichste Stelle" - c. m.)

E dopo che tutti gli ostacoli sono stati superati, ecco la difficoltà di giungere in cielo senza altri aiuti. Dante qui un poco estrapola, sospende il problema della forza di gravità. Semplicemente viene innalzato nella logica della fede. Dopo aver attraversato speditamente le ultime sfere oggi si direbbe la stratosfera, la ionosfera - ecco un ultimo mutamento di prospettiva. Una virata che torce ancora una volta lo spazio e il tempo, quello cristiano e quello greco-mitico e persino quello storico $[. .$.$] nella contrapposizione tra Terra e Cosmo.$

Siamo dinanzi a un inizio illogico e immemoriale che da secoli sfida interpreti, esegeti, pensatori con un'aporia insolubile, dove ciascun lettore è chiamato a ripercorrere le vicissitudini dell'ominazione. ${ }^{32} \mathrm{Nel}$ caso di Grünbein - gran viaggiatore e, soprattutto, appassionato esploratore di cosmi marini sommersi - la presenza del mare amplifica una passione profondamente intrecciata alla produzione poetica: ${ }^{33}$

Das ist die See, wie sie lebt. Sie feiert

Ende und Anfang einer jeden Geschichte, macht

Aus jeder Moderne eine Antike der Zukunft,

Aus jeder Antike die versunkenste aller Moderne. (“Studien in Aquamarin” 103)

Ecco il mare, la sua vita. Celebra

Fine e inizio di ogni singola storia, fa

Di ogni modernità una futura antichità,

di ogni antichità la più sommersa di tutte le modernità.

In questi versi, che chiudono la lirica sopra un ritmo di risacca, l'istanza poetante è esterna e interna. Nella strofa precedente, sgranati con grazia meravigliata, incontriamo gli abitanti degli abissi, tra cui il merletto ovvero la rosa di Nettuno («Neptunschleier»):

Gespensterkrabben weiden die Neptunschleier,

Die rosa Röhrchenschwämme nach Kleintieren ab.

In der Drift fächelt die Schraubensabelle

Der Seescheide Kühlung zu und dem Federwurm. (102)

32 Vorrei qui citare Barolini: «L'instabilità dell'analogia è strutturale, poiché il "punto solo" è analogo sia all'oggetto della visione, Argo, sia alla durata della visione, venticinque secoli. A rendere la terzina ancora più difficile da afferrare è il fatto che la sua azione principale è l'atto di dimenticare: un dimenticare attivo, continuo, che cresce senza soluzione di continuità. Infinitamente affascinante e suggestiva, impenetrabile e densa, e concettualmente e sintatticamente illogica (pur aprendo uno spiraglio per penetrare la dialettica tra simultaneità ed eternità, istante e durata, conflazione ed estensione), sicché nemmeno i versi finali del canto sono in grado di uguagliarne la capacità di unire il tempo in un "punto solo", l'analogia di Nettuno è un emblema perfetto della poetica di Paradiso XXXIII» (La "Commedia" senza Dio 345).

33 A titolo di esempio: "Zeit der Tiefseefische"; Die Bars von Atlantis; "Die Akademie des Meeres"; "Die Insel, die es nicht gibt" (562). La figura del palombaro di Delo in "Galilei vermißt ..." 97. Sulle immersioni in relazione a Descartes si veda Michael Eskin (2011) 401-402 nonché infra. Sulla dimensione orizzontale della traversata: Grünbein, "Dichtung als Schifffahrt", 16-17. 


\title{
Durs Grünbein. Un'idea di Dante 3D \\ Rosalba Maletta
}

Granchi fantasma brucano i merletti di Nettuno,

Le spugne tubolari rosate in cerca di animaletti.

Nella corrente di deriva ondeggia lo spirografo

Portando refrigerio all'ascidiaceo e all'anellide.

Reteporella grimaldii è un briozoo sciafilo della famiglia Reteporidae, su cui i granchi pascolano in cerca di piccoli animali: tutto un universo si anima. ${ }^{34}$ La vita corre verso gli inizi; achiropita, immota comincia mentre finisce. Nei recessi marini il poeta-subacqueo la contempla.

Se Nettuno emerge dalle acque lungo l'asse delle ordinate o, quantomeno, risale a pelo d'acqua per ammirare l'ombra d'Argo, sul piano delle ascisse la nave degli Argonauti solca $\mathrm{i}$ mari alla conquista dello spazio nella dimensione orizzontale che persegue espansione, dominio, conquista. ${ }^{35}$

La prima volta in cui il dio del mare prende atto che l'essere umano varca il suo regno, interviene sul tempo ciclico del mito istituendo una temporalità che procede lungo una retta con punti all'infinito:

Einmal vermessen läßt uns der Raum wunschlos zurück.

Langeweile, codiert, macht den Tod zur Null im Perfekt.

Ein neuralgischer Punkt, zwischen X und X auf dem Sprung

jagt sich das Ich nun, verstört, durch ein Fehlerprogramm.

("Dieu trompe-l'œil"19 - c. m.)

Una volta misurato, senza desideri ci lascia lo spazio.

Codificata, la noia rende la morte uno zero al passato prossimo.

$\mathrm{L}$ 'Io, un punto nevralgico in affanno tra $\mathrm{X}$ e $\mathrm{X}$,

orasi insegue stravolto attraverso un programma di errore.

Di conquista in conquista, di transito in transito l'umano è pervenuto alle grandezze scalari, ai vettori, ai diagrammi sino al rendering. Programmi specifici possono realizzare visualizzazioni dinamiche degli oggetti 3D che sono vere e proprie animazioni. Il monitor permette di aprire una finestra, una struttura inquadrante in cui l'obiectum appare secondo prospettive impensate. Nel nostro caso il monitor, la finestra inquadrante riposano nell'immaginazione creativa di Grünbein che rivisita Dante con gli occhi, la mente, il corredo linguistico, psichico, cognitivo, affettivo di un poeta del terzo millennio.

\section{Nachträglichkeit: I'ordine del poetico}

Consideriamo ora i versi di Paradiso XXXIII, che impegnano Grünbein almeno dal 2009, insieme alla terzina seguente:

\author{
Un punto solo m’è maggior letargo \\ che venticinque secoli a la 'mpresa \\ che fé Nettuno ammirar l'ombra d'Argo. \\ Così la mente mia, tutta sospesa, \\ mirava fissa, immobile e attenta,
}

34 Monaco Nature Encyclopedia: https://www.monaconatureencyclopedia.com/reteporella-grimaldii/ https://www.monaconatureencyclopedia.com/reteporella-grimaldii/.

35 Su questo aspetto cfr. Maletta, "Grünbein e Dante” 295-299. 


\section{Durs Grünbein. Un'idea di Dante 3D \\ Rosalba Maletta}

e sempre di mirar faceasi accesa. (Paradiso XXXIII, vv. 94-99)

Nel chiosare la strofa, che tanto lo affascina, nel 2009 Grünbein ricorre per due volte alla figura di paragone sia sul piano del significante che su quello del contenuto:

Es legt den Vergleich zweier Zeiten nahe, die an sich unvergleichbar sind: die des auf ewig angehaltenem Atems imMoment der mystischen Einheit mit Gott — und jene andere lineare, die Zeit der Historiker, in der das Rad erfunden wird und der Webstuhl, das Schiff und der Buchdruck und eines Tages auch Flugzeug und Mondrakete. Dantes Vergleich, so die eine Lesart, geht hier deutlich zuungusten der letzteren aus, so als wollte er sagen: In einem Moment der Unachtsamkeit gegen Gott ist mehr verloren, als wenn der Mensch ein paar Jahrhunderte lang den Fortschritt verschlafen hätte. Es ist aber Neptun, der hier ins Staunen gerät, nicht der Mensch, und schon darum sind weitere Lesarten gefragt. Oder einladender ausgedrückt: Es braucht immer neue Leser für ein Gedicht von solch verborgener Komplexität. ("Meerfahrt mit Dante" 43 - c. m.)

Si suggerisce il paragone tra due temporalità in sé incomparabili: quella del respiro, trattenuto in eterno nel momento dell'unione mistica con Dio - e quell'altra, lineare, l'epoca degli storici, in cui si inventò la ruota e il telaio, l'imbarcazione e la stampa e un bel giorno anche aeroplano e sonda lunare. Il paragone di Dante, così recita un'interpretazione, va qui nettamente a svantaggio della seconda, come se egli volesse dire: In un momento di disattenzione verso Dio si perde di più che se l'essere umano avesse dimenticato il progresso per un paio di secoli. E tuttavia qui a stupirsi è Nettuno, non l'essere umano, e già per questo sono necessarie ulteriori interpretazioni. Ovvero, formulato in maniera più stimolante: c'è bisogno di sempre nuovi lettori per un poema di una tale recondita complessità.

Incontriamo nuovamente quel modo di avanzare per slittamenti comparativi e letture trasversali che Grünbein individua quale elemento caratterizzante la Divina Commedia. Procedimento insaturo e insaturabile, esso mette in moto l'energia creativa secondo costellazioni inconsapevoli e un'emersione in superficie controllata, che molto ci interessa rispetto alla resa del 2021 e che trova riscontro nel metodo freudiano. ${ }^{36}$

L'impressione è che il poeta proprio qui si aspettasse, almeno dal 2009, in quella dimensione di futuro nel passato che la psicoanalisi denomina Nachträglichkeit. Qui egli si aspetta in base al metodo che propone per la lettura dei testi poetici — i propri e gli altrui — da cui ha preso le mosse il presente lavoro:

Seit Lucretius und Dante, Bruno und Leonardo war seinesgleichen [in quanto poeta, RM] dabei zu sichten, was an Zeichen, an Alphabeten über die Erde verstreut lag. Heute nun, unterwegs auf einer in vielerlei Sprachen verstummenden Erde, ist es sein zweites Analphabetentum, sein modernes Selbstbewußtsein, Transformator von Sprachen zu sein, die vielleicht zu verengen drohen. Neu, wirklich neu ist sein Versuch, sich entlang der semantischen Wundränder vorzutasten und in sich selbst, in seinem noch staunenden Grauen, von außen kommend all den Bewegun-

36 «In der Psychoanalyse können wir nur mit Hilfe von Vergleichungen beschreiben. Das ist nichts Besonderes, es ist auch anderwärts so. Aber wir müssen diese Vergleiche auch immer wieder wechseln, keiner hält uns lange genug aus" ("Die Frage der Laienanalyse" 222) - «In psicoanalisi possiamo descrivere solo mediante il ricorso ai paragoni. Nulla di speciale, così si procede pure in altri ambiti. Tuttavia siamo sempre costretti a variare questi paragoni, nessuno di essi regge abbastanza a lungo». 


\section{Durs Grünbein. Un'idea di Dante 3D \\ Rosalba Maletta}

gen von Zivilisation und Natur zuwiderlaufen, die ihn von früh an als Brüche markieren. (“Ameisenhafte Größe” 15) 37

Dai tempi di Lucrezio e Dante, Bruno e Leonardo, furono quelli come lui a individuare e vagliare i segni e gli alfabeti sparsi sulla terra. Oggi, in viaggio in un mondo che sta mettendo a tacere un gran numero di lingue, sono il suo secondo analfabetismo, la moderna autoconsapevolezza di fungere da trasformatore di linguaggi a correre forse il rischio di ridursi. Nuovo, davvero nuovo, è il suo tentativo di farsi strada lungo i bordi della ferita semantica e di contrastare in se stesso, nel suoorrore ancora attonito, dall'esterno, tutti i movimenti della civiltà e della natura che sin dal principio lo segnano come violazioni.

\section{Argo 3D}

Qui si consuma - da trasformatore a trasformatore - il passaggio di testimone tra Dante poeta e Grünbein poeta nonché assiduo lettore di Dante:

Ein wenig Schlafsucht bringt mich mehr ins Arge als zweieinhalb Jahrtausende zuvor der Schatten, der Neptun staunen ließ: die Heldenfahrt der Argo.

Nel rendering del 2021 non è più presente il lessema "punto", assorbito in "ein wenig", quantificatore indefinito dello stato letargico del pellegrino dinanzi alla vista inaudita, ora espresso con il composto «Schlafsucht».

Grünbein crea una rispondenza chiastica tra Schlafsucht della prima riga e Schatten della seconda. Più che la impresa è l'ombra che questa produce nelle acque a far meravigliare (staunen) Nettuno.

La presenza insistita delle spiranti alternate - ora sorde ora sonore, forti ovvero leni, forma all'interno della terzina un legato che - rinforzato dalla prevalenza della vocale aperta prepara l'emersione del significante Argo che rima con la locuzione ins Arge.

La stupefazione del dio marino - sino a allora signore incontrastato delle acque - è ben sostenuta sul piano fonosimbolico, della struttura materica e della superficie fonemica, da quelli che Contini definirebbe «valori fonici puri» ("Esercizio di interpretazione" 27).

Sul piano espressivo paradigmatico la locuzione ins Arge ${ }^{38}$ chiama in causa il demonio (al maschile Der Arge) proprio nel momento della massima eclissi dell'Io consapevole (Schlafsucht) nel compimento della visione. "Ins Arge" assona del resto con "Argo" nella polisemia significante. Etimologicamente ó $\rho \gamma o ́ \varsigma$ vale "splendente"; "luccicante", ma anche "rapido"; "veloce". ${ }^{39}$ In qualità di nome proprio Argo di Tespi costruì l'omonima nave degli Ar-

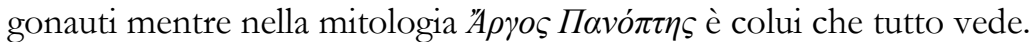

"Die unheimlichste Stelle" dispiega il potenziale che inerisce alla visione poetica in grado di superare le coordinate spaziotemporali di una realtà cristallizzata nella «partizione del sensibi-

${ }^{37}$ La prosa Ameisenhafte Größe è volta a un'assunzione di responsabilità poetica che è etica del linguaggio. Nell'intervista rilasciata a Ruzzenenti Grünbein riprende la medesima figura di trasformatore per Dante che è già presente in questa riflessione poetica e poetologica degli anni Novanta.

38 DUDEN: arg come aggettivo e le locuzioni attinenti: https://www.duden.de/rechtschreibung/arg (consultaz. 18.08.2021). Si veda pure il sostantivo das Arg per lo più in frasi dove è accompagnato da una negazione quale sinonimo di falsità, malevolenza, intento di portare nocumento: https://www.duden.de/rechtschreibung/Arg (ultima consultazione 18.08.2021).

39 Per la voce ó $\rho \gamma o ́ \varsigma$ cfr. Chantraine, pp. 104-105. Etimologicamente Chantraine pone all'origine del termine una nozione che esprime il bianco abbagliante del fulmine insieme alla velocità (104). 


\section{Durs Grünbein. Un'idea di Dante 3D \\ Rosalba Maletta}

le» (Rancière) per congiungersi a quella dimensione, dove l'ispirazione domina incondizionata. La passione di Grünbein per le origini, per Nettuno e il suo regno, per Omero e Ovidio, per Dante e Shakespeare non si risolve in una disputa tra Cancel Culture e Trigger Warning che, in epoca di memorie collettive standardizzate, pretende di dettare quote di oblio e ricordo eguali per tutti:

Ich bin überzeugt davon, daß wir die Antike allein deshalb für die Zukunft benötigen, damit die Menscheit nicht in tausend kleine Erzählungen zerbricht. Ich wehre mich vehement dagegen, dass (sic!) man glaubt, im Zuge der kompletten Entkolonialisierung des Geistes - auch des europäischen - nun auch noch die Antike über Bord werfen zu sollen. Gleichsam als wäre die eine Altlast. ${ }^{40}$

Sono convinto che abbiamo bisogno dell'antichità per il futuro, se non altro perché l'umanità non si frantumi in mille piccole narrazioni. Mi oppongo fermamente all'idea che nel corso della completa decolonizzazione dello spirito, compreso quello europeo, si debba ora gettare a mare anche il mondo antico. Come se fosse un vecchio fardello.

La filiazione in cui Grünbein si riconosce, si autorizza (auctoritas) anche nel senso del rendering dell'idioma del padre delle lettere mondiali in quello di uno dei suoi eredi che lo traspone in una lingua lontana per ceppo e provenienza. Il rendering, le traslazioni, l'operato e la linea genealogica che la poesia e le riflessioni grünbeiniane ci presentano, inducono a cercare risposte. Risposte che non spengano la voce dei poeti, lo stupore orrificato di Nettuno e il canto delle imprese dell'umano.

Che Grünbein abbia da considerarsi un classico dei nostri tempi risulta dalla sua capacità di rileggere le nostre origini e di figurarsi un'Europa unita ${ }^{41}$ che sicuramente sarà migliore per i figli dei figli, per chi ha esperito il regime poliziesco e il controllo sui corpi, l'intrusività e la delazione che deformano la mente, distorcono le parole e fanno implodere la pelle psichica.

In una situazione di questo genere le sensazioni corporee, i percetti e gli Affekte espressi dalla voce poetica liberano una tensione che è ricerca etica: «Schreiben war immer dann am lebendigsten, wenn es sich an den bloßliegenden Nerven vorantastete, beweglich in jede Richtung, wachsam und sensitiv» - «Lo scrivere espresse sempre il massimo della vitalità, quando cercò di farsi strada attraverso i nervi scoperti, mobile in ogni direzione, vigile e sensitivo» ("Reflex und Exegese" 65-66).

Nel 2021 Grünbein decide di trasferire nella propria lingua, forse e per dir meglio, nel bagno di suoni che lo impregna da prima della nascita, il nucleo più intimo del significante Heim, costitutivo dello Un-heim-liches. Non è la funzione fatica del linguaggio a rivelare il poeta a se stesso. La messa a nudo della lingua definisce linee, punti, ritmi e coordinate di un dire che è un bene dire:

Wie komme ich meiner eigenen Rede auf die heimlichen Schliche? Wenn ich weiß, was auch alle anderen wissen - - warum mich dann mitteilen?

Ich mißtraue mir - das ist der Anfang. Ich suche nach einer Lösung im Ausdruck durch den Sprung in die Dichtung. Es ist die Sprache als Basis, die mich in ihrem poetischen Eigensinn mir enthüllt. ("Das Punktum des Gedichts" 96)

40 Così il poeta risponde alle sollecitazioni di Marek Gross nella conversazione per le strade di Roma ("In den Straßen Roms") che il critico pone in appendice al suo volume (p. 226). Su questo punto si veda pure: Grünbein, Vom Stellewert der Worte 41-42.

${ }^{41} \mathrm{Si}$ veda il Discorso Europa, die Insel, die es nicht gibt, tenuto da Grünbein a Llubljana (Slowenien) alla fine di agosto del 2021, testo ancora inedito gentilmente fornitomi dall'autore in data lunedì 2 agosto 2021. Si veda pure Eskin, "Durs Grünbein and the European Tradition". 


\section{Durs Grünbein. Un'idea di Dante 3D \\ Rosalba Maletta}

Come scopro i trucchi reconditi del mio stesso discorso? Se so quel che anche tutti gli altri sanno, a che pro allora prendere la parola?

Diffido di me stesso - questo è l'inizio. Cerco una soluzione per esprimermi attraverso il salto nella poesia. È il linguaggio come base che, nella sua ostinazione poetica, mi rivela a me stesso.

Forse il passaggio oltremodo inquietante consiste proprio in questa autorivelazione mediante il linguaggio:

L’uomo non preesiste al linguaggio, né filogeneticamente né ontologicamente. Non è possibile raggiungere uno stato in cui l'uomo sia separato dal linguaggio, che egli elaborerebbe per 'esprimere' quanto avviene in lui: è il linguaggio a informare la definizione dell'uomo, e non il contrario. (Barthes 14)

Come se per anni Grünbein fosse rimasto in ascolto di quel che Barthes chiama le bruissement de la langue (79): della sua propria linguae di quella di Dante che trasfonde gli echi della parlata di Firenze nel poema che va componendo nello «scendere e l' salir per l'altrui scale» (Paradiso XVII, v. 60).

\section{Dante lux aeterna del moderno}

In un saggio dedicato a Broch, Ezio Raimondi rileva come nella terza e ultima cantica Dante sia «di continuo impegnato in operazioni straordinarie dentro la spazialità nuova, abbagliante del Paradiso, dove non c'è più la notte ma una specie di giorno perenne». Attraverso la lettura di un contemporaneo, nel caso di Raimondi Hermann Broch, nel nostro caso Grünbein, «si dimostrano le vitalità degli antichi, i loro germogli» (61).

A più riprese, nella conversazione con Heinz-Norbert Jocks, Grünbein sottolinea come il lessema "moderno" acquisisca nella sua opera e nella sua poetica una valenza sovratemporale. Dante è moderno nella misura in cui rompe con l'universo rappresentazionale preesistente:

Entscheidend ist der Entwicklungssprung, der Griff über den Kanon der eigenen Zeit hinaus. Modern ist, was Zukunft hat, es zeigt sich an seiner dauernden Wiederkehr. Nicht im Sinne Nietzsches, als Wiederkehr des Gleichen, sondern als zyklischen Auftauchens des Neuen. Dieses Bewegunsprinzip läßt sich nicht für die Neuzeit reservieren. (Grünbein; Jocks 16-17)

Decisivo è il salto evolutivo, la presa oltre il canone del proprio tempo. Moderno è ciò che ha futuro, si manifesta in un continuo ritorno. Non nel senso di Nietzsche, come ritorno dell'identico, bensì quale ciclico affiorare del nuovo. Questo principio dinamico non può essere riservato ai tempi moderni.

Tale circostanziata lettura del Moderno libera un fulcro di ascendenze e discendenze di natura prettamente spirituale:

Moderne ist, nach meiner Auffassung, ein Phänomen des Ungleichzeitigen, ein Kreuzungspunkt vieler unzusammenhängenden Progressionen zu verschiedenen Zeiten, von Entwicklungssprüngen, die nichts miteinander gemein haben als den einen Effekt, über ihren Anlaß hinauszuschießen in eine überzeitliche Sphäre. In dieser Sphäre sind Leute wie Archimedes und Einstein Zeitgenossen. (Der cartesische Taucher 11-12)

La modernità è, a mio avviso, un fenomeno di squilibrio, un punto d'intersezione di molte progressioni incoerenti in epoche diverse, di salti evolutivi che non hanno nulla in comune se non il solo effetto di proiettare al di là della loro occasione in una sfera ultratemporale. In questa sfera, individui come Archimede e Einstein sono contemporanei. 


\section{Durs Grünbein. Un'idea di Dante 3D \\ Rosalba Maletta}

Quel che colpisce il poeta del terzo millennio è come la voce di Dante - che nelle tre cantiche riecheggia in miriadi di timbri, colori, sfumature - abbia saputo raccogliere l'essenza più intima del volgare che andava formandosi e che, dopo di lui, non sarà più lo stesso:

D. G. - Die Annäherung an das Genie der Sprache. Er hat nur sie, die Sprache in seiner Verbannung und schöpft alle ihre Möglichkeiten aus, verschafft sich maximales Gehör. Er ist das Kind, das in der Muttersprache badet und alle Vokale und Konsonanten bis an die Grenzen ausschöpft. Er gab der Sprachkunst die Regeln auf so unerbörte Weise, daß alle Sprachteilnehmer vom selben Stamm, alles Italienisch sprechende Volk fortan durch das von ihm errichtete Portal gehen mußten. (Grünbein; Ruzzenenti - c. m.)

D. G. - L'accostarsi al genio della lingua. Non ha che questo nell'esilio, il linguaggio, e ne sfrutta tutte le possibilità, procurandosi il massimo dell'ascolto possibile. È il bambino che fa il bagno nella lingua materna e sfrutta tutte le vocali e le consonanti fino al limite. Impartì all'eloquenza le regole in maniera tanto inaudita che tutti i parlanti la lingua dello stesso ceppo, tutti gli italofoni da allora in poi dovettero passare attraverso il monumentale portale che egli aveva eretto.

Indelebile impronta del singolo, anche nella Commedia la voce è un fenomeno liminare e come tale trattata da Dante. ${ }^{42}$ Non solo corpo e neppure solamente spirito, non sensuosità e nemmeno solo senso, essa veicola l'Affekt mediante timbro, risonanze e colore senza trascurare l'intendimento dell'intelletto, il linguaggio e l'immagine che a esso si accompagna:

D. G. - Diese Verse kann man durchaus vor sich hin sprechen auch ohne nähere Italienischkenntnisse, sie funktionieren als Klangstruktur. Es rundet sich, es gibt überraschende Lautsprünge, Assonanzen und teuflisch onomatopoetische Stellen. Da ist das große System der Vokalabstimmungen, eine Chromatik wie in musikalischen Kompositionen. Und ein schwindelerregendes Denken in Bildern. Pounds berühmte Dreikomponentenlehre der Poesie - melopoeia, phaenopoeia, logopoeia - ist wie auf Dante zugeschnitten. Dante wäre das Schulbeispiel. Hinzu kommt das in allem waltende Prinzip der Allegorie und der biblischen Ikonographie. Metaphern und Signifikaten gleiten wie Weberschiffchen hin und her. Die italienische Ausgabe der Commedia, die ich mir irgendwann zulegte, war so stark annotiert und zeilenweise kommentiert, dass man sich wie in einem Gestrüpp verfing. Vorsicht, war das Signal, hier hat alles einen doppelten Boden. (Grünbein; Ruzzenenti)

D. G. - Questi versi si possono pronunciare tra sé e sé anche senza una conoscenza approfondita dellitaliano, funzionano come una struttura sonora. A completare il tutto, ci sono sorprendenti salti di suono, assonanze e passaggi dannatamente onomatopeici. C'è il grande sistema delle accordature vocaliche, un cromatismo come nelle composizioni musicali. E un pensiero vertiginoso in immagini. La famosa teoria della poesia a tre componenti di Pound - melopoeia, phaenopoeia, logopoeia - pare fatta su misura per Dante. Dante sarebbe l'esempio da manuale. Inoltre, c’è il principio dell'allegoria e dell'iconografia biblica che prevale in tutto. Metafore e significati scivolano avanti e indietro come navette per la tessitura. L'edizione italiana della Commedia, che a un certo momento mi comperai, era così fittamente annotata e commentata rigo per rigo che era come rimanere impigliati in un groviglio. Il segnale era: attenzione, tutto qui ha un doppio fondo.

${ }^{42}$ Cfr. Contini, Un'idea di Dante. Sulle variazioni e gli adeguamenti della voce di Dante che si approssima all'Eden si vedano: Singleton, La poesia della Divina Commedia, in particolare Parte II e III (133-564); Barolini, La "Commedia" senza Dio, in particolare capp. 7-10 (199-347). 


\section{Durs Grünbein. Un'idea di Dante 3D \\ Rosalba Maletta}

La riflessione che ci porge Grünbein riguarda il ricorso alla retorica ma investe appieno un immaginario filtrato alla luce di una soggettività potente, non già malgrado bensì proprio grazie all'uso personale dell'allegoria, del simbolismo, dell'iconografia biblica. ${ }^{43}$

\section{La poesia tra "Heldenfahrt" e "Heidenangst"}

Chiediamoci perché nella trasposizione del 2021 Grünbein ci faccia entrare nella sua idea di Dante sostituendo al lessema punto l'avverbio di quantità indeterminata "ein wenig" e al sostantivo più aulico e astratto "Entrücktheit" il composto "Schlafsucht", più realistico e legato alla corporalità.

La soggettività, che osserva il mondo globalizzato nel pieno della rivoluzione digitale, riconosce la struttura dell'immagine-suono in questione e la riordina secondo una sintassi, una ritmica e una cadenza che derivano dalle coordinate storico-geografiche della cultura e della lingua di appartenenza ma pure da un codice poetico che la voce di Grünbein ha acquisito nel corso degli anni e non cessa di rielaborare.

È una struttura d'immaginazione su cui la lingua ritaglia priorità che l'ingegno ha individuato e cullato. L'immagine-suono di partenza nella mente, nell' anima, nella lingua del poeta subisce incessanti modificazioni come la vita senziente, intellettiva, cognitiva e inconscia.

Basandosi su una paretimologia, un paragramma resi possibili dalla lingua materna, in quella peculiare forma di rendering - che chiameremmo poetico e che Grünbein anima e accende nella nostra psiche - Argo rima con ins Arge. Nella resa del 2021 Grünbein rispetta la rima rovesciandone senso e funzione.

All'impresa eroica ("Heldenfahrt") risponde in epifora un significante che fa segno al danno, a qualcosa di negativo, sinanco di demonico. ${ }^{44}$

La condizione dell'essere umano deriva dalla nudità biologica ("Brief über den Sarkasmus und das Gedicht als Konzept" 52-53). Il fatto che, in quanto vivente, l'umano sia esposto alla dissociazione, ne fa la maschera di un Io transeunte, vano e caduco. Spinge a cercare appigli in ciò che è tipico, conforme, uniformante. La poesia persegue una via individuale, esprime una soggettività unica e irripetibile, intesse trame e intreccia orditi al singolare plurale proprio a partire dalla fragilità umana (Ivi).

Nel saggio "Warum schriftlos leben" (1999) Grünbein dichiara che la motivazione alla scrittura sorge da un'angoscia feroce, in tedesco "Heidenangst", ${ }^{45}$ nei confronti del Nulla onnipresente sia in senso temporale che spaziale (61 ss).

Se assumiamo, con Lacan, che l'angoscia è ciò che non inganna, la via che lo scrittore individua nei suoi resoconti in versi e in prosa sul mestiere del pensare e trasmettere immagini del pensato, è proprio «ciò che non inganna».46

${ }^{43}$ Su questi aspetti della Divina Commedia cfr. Singleton e Barolini nonché il finissimo Ossola (96-121 e passim).

44 DUDEN ONLINE: arg come aggettivo e le locuzioni attinenti: https://www.duden.de/rechtschreibung/arg (consultaz. 18.08.2021). Si veda pure il sostantivo das Arg per lo più in frasi dove è accompagnato da una negazione quale sinonimo di falsità, malvagità, intento di portare nocumento: https://www.duden.de/rechtschreibung/Arg (ultima consultazione 18.08.2021).

45 DUDEN: «Heidenangst: umgangssprachlich emotional verstärkend; sehr große Angst vor jemandem, etwas» https://www.duden.de/rechtschreibung/Heidenangst (ultima consultazione 18.08.2021). 46 «[...] è proprio sul lato del reale, in prima approssimazione, che dobbiamo cercare ciò che, nell'angoscia, non inganna» (Il seminario. Libro X 187). 


\section{Durs Grünbein. Un'idea di Dante 3D \\ Rosalba Maletta}

La poesia sussiste in quella scanalatura capace di porre in rispondenza chiastica $i$ due composti "Heldenfahrt" e "Heidenangst". Entrambi lottano per la conquista di uno spazio interno che urge e preme per proiettarsi all'esterno. Entrambi propongono una risposta agli enigmi di Descartes. A più riprese il «Dante della teoria della conoscenza» (Der cartesische Taucher 22) si chiede come sia possibile che qualcosa di immateriale come la mente entri in relazione, addirittura riesca a "guidare" la sostanza materiale di un corpo. E ancora: come possiamo entrare in relazione non solo con la nostra mente ma addirittura con la mente di altri?

Considerando la resa di "letargo" non già con "Entrücktheit" bensì con il più prosaico e fisiologico composto "Schlafsucht" e passando in rassegna le innumerevoli visioni che costellano la Commedia, incontriamo i dubbi e le domande che istituiscono la soggettività moderna.

E se per Descartes è indispensabile porre un discrimine tra vero e verisimile quest'ultimo da intendersi come qualcosa di talmente simile al vero da costituire l'inganno supremo - ricordiamo quanto i personaggi della Commedia, Dante stesso come agens e auctor, siano impegnati a fingere di non fingere. ${ }^{47}$ I molteplici sviamenti, i "depistaggi" messi in atto nel poema sono volti a sostanziare «quel ver c'ha faccia di menzogna» (Inferno XVI, v. 124).48

Possiamo a questo punto forse ipotizzare che la Schlafsucht proposta da Grünbein nel 2021 sia il miglior antidoto alla Heidenangst. Quest'ultima attanaglia lo scrittore dell'epoca digital-globale poiché l'inganno massimo diviene la poesia degli affetti, delle emozioni, la celebrazione dell'immaginazione creativa che proietta la caducità, il fatto che la vita sia biologicamente condizionata, in un'eternità di scambi:

Tu dici: "Io veggio l'acqua, io veggio il foco,

l'aere e la terra e tutte lor misture

venire a corruzione, e durar poco" (Paradiso VII, vv. 124-126)

Così Beatrice traduce le perplessità e i dubbi di Dante rispetto all'elemento transeunte in relazione alla salvazione. Ed è sempre Beatrice e la beatrice che, nel primo Canto del Paradiso, istruisce il pellegrino, finalmente giunto alla meta agognata:

Ne l'ordine ch'io dico sono accline

tutte nature, per diverse sorti,

più al principio loro e men vicine;

onde si muovono a diversi porti

per lo gran mar de l'essere, e ciascuna

con istinto a lei dato che la porti. (Paradiso I, vv. 109-114)

Il fiuto poetico di Dante whicheverman (Singleton) ${ }^{49}$ non cessa di mostrarci che ne è venuto da quella sete di conquista che non cessa di motivare gli Argonauti presenti, passati e futuri.

${ }^{47}$ Su questo aspetto: Singleton, “The irreducible Dove” (129) nonché "Elementi di struttura” (88).

$48 \mathrm{Su}$ questo punto, legato al tema della verisimiglianza e della simulazione, si veda Barolini, Il miglior fabbro, in particolare cap. 3 "Risoluzione epica" (153-226), soprattutto 171 ss. E più recentemente: Il secolo di Dante. Viaggio alle origini della cultura letteraria italiana, cap. 8 (245-265), soprattutto 250-251.

49 «Nell'allegoria, il viandante è qualsiasi cristiano: è l'bomo viator, ma, a rigor di termini, non è Ogni Uomo (Everyman). È piuttosto "Qualsiasi Uomo" (Whicheverman); chiunque, cioè, possa essere scelto per questo viaggio a Dio, mentre vive ancora in questo mondo, dove tutti, volenti o nolenti, siamo viandanti. [...] Che tale viaggio bic et nunc sia una possibilità aperta a tutti, resta il postulato fondamentale e, per Dante, la dottrina su cui egli può costruire l'allegoria della Commedia. Se il protagonista del viaggio riflesso è "chiunque", il tempo corrispondente sarà allora "quandunque". Molti hanno intra- 


\section{Durs Grünbein. Un'idea di Dante 3D \\ Rosalba Maletta}

Per via di intrecci intertestuali Grünbein coglie appieno il messaggio e ci fornisce una chiave di lettura di Paradiso XXXIII, vv. 94-96 che si delinea come un viatico per i nostri tempi immersi nel world wide web.

\section{Passaggio di testimone}

Se è Descartes - con le assonometrie e le proiezioni ortogonali - a dare senso allo spazio e alle identificazioni proiettive del soggetto nella res extensa, è Dante a precederlo e anticiparlo nella capacità di animare la res cogitans. L'umana finitudine si misura sulle coordinate della storia del mondo e della salvazione di un'anima. Le visualizzazioni di Dante e Descartes sono tentativi, ciascuno lungo il proprio linguaggio, di dare una funzione ordinatrice al caos che è l'umana esistenza e che invade ogni singola vita.

Nella Commedia è la dimensione soggettiva a fare la differenza, a creare un capolavoro senza precedenti:

D. G. - Aus der Commedia kann man Dantes Bibliothek zusammenstellen, da wird nichts versteckt. Der entscheidende Effekt ist aber die Stimme des Dichters - alles erscheint im hohen Maße subjektiviert, persönlich und eben auch kritisch angeeignet, das ist das Moderne daran. Wie bei Ovid, der die griechischen Mythen und ihre römische Adaption alle noch einmal durch einen persönlichen Filter schickt und sie damit gleichsam psychologisiert. Das eigentliche Vorbild war aber Vergil, den hat er studiert so wie wir Späteren dann Dante studiert haben. Vergil ist der Führer, dem er sich anvertraut, mit dem er durch das Totenreich geht als ein Kind seiner Zeit, bis er die Hand loslassen muß. Wir hören Dante, wir hören diesen einen, historisch äußerst facettenreich überlieferten Menschen aus Florenz, den Sohn seiner Stadt, und zugleich hören wir abertausende andere Stimmen. Wir hören bis in den Tonfall genau ihre Meinungen, ihre Charakteristik, ihr Temperament, ihre Launen und scharfen Urteile. Wir begreifen Aspekte des gemeinsam Biographischen, und das schafft eine Atmosphäre der Authentizität, die wiederum aufregend modern ist, als hätten wir es mit einem Zeitungsleser aus dem 13. Jahrhundert zu tun. (Grünbein; Ruzzenenti).

D. G. - Si può mettere insieme la biblioteca di Dante dalla Commedia, non c'è nulla di celato. L'effetto decisivo, tuttavia, è la voce del poeta - tutto appare in larga misura dal punto di vista soggettivo, personale e, appunto, anche critico, questa è la sua modernità. Come nel caso di Ovidio, che trasmette i miti greci e il loro adattamento romano attraverso un filtro personale, per così dire psicologizzandoli. Ma il vero modello era Virgilio, che egli studiò proprio come poi noi studiammo Dante. Virgilio è la guida a cui si affida, con la quale cammina nel regno dei morti come un figlio del suo tempo, fino a quando non deve lasciargli la mano. Ascoltiamo Dante, udiamo questo solo, unico fiorentino, che la storia ci ha tramandato in maniera grandemente sfaccettata, figlio della sua città, e allo stesso tempo ascoltiamo migliaia e migliaia di altre voci. Cogliamo sin nella cadenza e intonazione le loro opinioni, le loro caratteristiche, il loro temperamento, i loro stati d'animo e i loro giudizi taglienti. Comprendiamo aspetti della biografia comune e questo crea un'atmosfera di autenticità che è a sua volta entusiasmante e moderna, come se avessimo a che fare con un lettore di quotidiani del XIII secolo.

Il passaggio di testimone struttura un gioco di staffetta in cui il poeta - immerso nel reale digitalizzato, dove è indispensabile fingere di fingere - denunzia il simulacro ${ }^{50}$ che copre

preso questo itinerarium mentis in passato, molti continueranno a intraprenderlo fino alla fine dei tempi» (Singleton, La poesia della "Divina Commedia"139-140).

50 «e simulacre n'est jamais ce qui cache la vérité - c'est la vérité qui cache qu’il n’y en a pas. Le simulacre est vrai» (Baudrillard 9). 


\section{Durs Grünbein. Un'idea di Dante 3D \\ Rosalba Maletta}

l'Ultra Nulla senza rinunziare a quella pratica etica del pensare che la poesia è ancora in grado di trasmettere, trasferire e trasformare proprio perché sfugge alle logiche consumistiche espresse da una quotidianità anestetizzata:

Niemals verliert, niemals vergißt sich

Der erste Schock, Atem

Solange es atmet, Denken

Solange es denkt. Ordnungen wechseln,

Taghimmel, Moden,

Landschaften kommen und gehen

Wie Sterne und das Gerede um Nichts

Regeneriert sich im Schlaf.

Sprache ist Rache des Fleischs

Durch den Kehlkopf. ("Ultra Null” 139)

Giammai si perde, giammai si dimentica

Il primo shock, respiro

Finché respira, pensiero

Finché pensa. Gli ordinamenti mutano,

I cieli diurni, le mode,

I paesaggi vanno e vengono

Come le stelle e le dicerie sul Nulla

Si rigenera nel sonno.

Il linguaggio è vendetta della carne

Attraverso la laringe.

Il linguaggio è irriducibile al simbolico, deve attraversare il corpo per giungere a compimento e nel momento in cui pronunzia e scrive questo corpo caduco, si eccede. Crea il suo proprio oltre come per Nettuno e il poeta che lo guarda dal fondo delle acque, mentre ricopre tutte le posizioni e attraversa tutte le ere geologiche:

Die Dichterseele gleicht dabei dem bereits erwähnten Flaschenteufelchen, wenn sie unterm

Druck der Vernunft auf und ab tanzt. Sie ist der cartesische Taucher, der zuletzt doch immer wieder an die Oberfläche der Erscheinungen zurückfindet. Dort, dicht unter der Gummihaut, die ihr Refugium von dem wahren, unermeßlichen Außenraum abtrennt, ist ihr liebster Aufenthaltsort. An dieser Stelle fühlt sie sich zirka siebentausend Jahre zu Hause. (Der cartesische Taucher 90-91)

Quando danza su e giù, sotto la pressione della ragione, l'anima del poeta somiglia al succitato diavoletto nella bottiglia. È il sommozzatore cartesiano che alla fine ritrova sempre la strada verso la superficie dei fenomeni. Il suo domicilio preferito è proprio là, sotto il rivestimento in gomma che separa il suo rifugio dallo spazio esterno, reale e incommensurabile. In questo spazio essa si sente a casa da circa settemila anni.

Il poeta sommo e il filosofo dotato di una spiccata inclinazione per les espaces imaginaires, sgranano immagini e metafore lungo i nastri trasportatori della nostra immaginazione: 


\section{Durs Grünbein. Un'idea di Dante 3D \\ Rosalba Maletta}

Dante lesen, heißt eine lange Wanderung unternehmen. Der Dichter-Erzähler und sein Vers, die geschmeidige Terzine, dieses unaufhörlich rollende Transportband, sind immerfort in Bewegung, selbst da wo sie Halt machen in Betrachtung, Rückblick und Gespräch. Man liest, hält kurz inne und wird sofort weitergezogen. ("Die unheimlichste Stelle")

Leggere Dante significa intraprendere una lunga peregrinazione. Il poeta-narratore e il suo verso, la duttile terzina, questo nastro trasportatore che scorre incessantemente, sono sempre in movimento, anche quando fanno una sosta per contemplare, per volgersi indietro e per conversare. Leggiamo, facciamo una breve pausa e subito siamo spinti oltre.

Viene in mente l'operazione di Douglas Hofstadter che in Le Ton Beau de Marot (531-540) ricostruisce il diagramma della terzina dantesca per mostrare proprio questo carattere di nastro continuo che la lanterna magica di una visionarietà senza precedenti e un orecchio finissimo fanno scorrere incessantemente: ${ }^{51}$

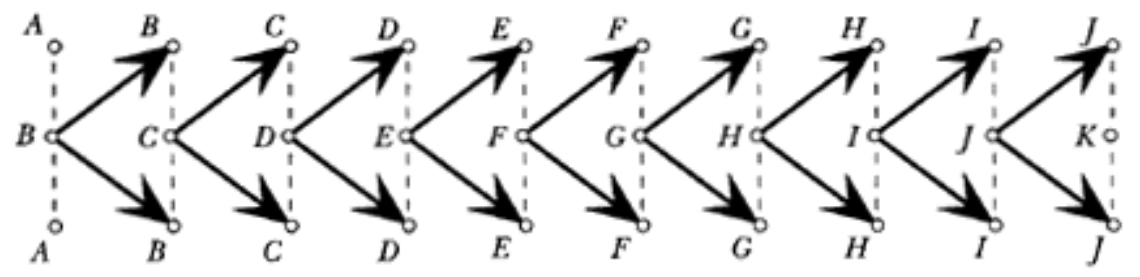

Se il principio del Medioevo era, nella scia dei classici latini, prodesse et delectare, con Virgilio e l'Eneide, con Stazio e la riscrittura delle genealogie nella rivisitazione della Tebaide e dell'Achilleide, Dante si inscrive in un lignaggio che egli stesso si è creato per via di immaginazione e filiazione poetica. Lo stesso vale per Descartes, iniziatore del soggetto che si sa finito e si rivela attraverso i sogni e l'algebra.

Benché supporto e matrice siano mutati, il diavoletto cartesiano non cessa di tornare a galla. Le coordinate di Monsieur Descartes giungono sino al rendering con cui Grünbein traghetta Dante nell'idioma succhiato con il latte materno immettendolo nell'era globale.

Dal notes magico di Freud all'oggetto 3D la dimensione immaginifica, le emozioni e le percezioni che i poeti proiettano sul mondo dal vertice di una singola esistenza non cessano di incontrare "die unheimlichste Stelle", inesausto processo in cui il passaggio di testimone assurge a quella dimensione etica della scrittura capace di riscattare un'epoca:

Indem das Gedicht dem zeitlichen Ablauf Hindernisse entgegenstellt, seien es metrische Barrieren, seien es lexikalische Entfernungen, sichert es sich seine Dauer, im Gedächtnis der Nachgeborenen ebenso wie im Erlebnishaushalt des einzelnen. Die Resistenz, die das Gedicht gegen die Zeit aufbringt, ist um so größer, je souveränerer das Realitätsprinzip (der Physik, des Verstandes, der phänomenalen Ordnung) außer Kraft gesetzt wurde. ("Mein babylonisches Hirn" 27)

Ponendo ostacoli allo scorrere del tempo, siano essi barriere metriche o distanze lessicali, ogni singola poesia si assicura la propria durata nella memoria dei posteri così come nel bilancio esperienziale dell'individuo. La resistenza della poesia al tempo è tanto più grande quanto più efficacemente è stato abolito il principio di realtà (della fisica, dell'intelletto, dell'ordine fenomenico).

51 Interessante l'operazione di Hofstadter di riportare il diagramma della terzina dantesca per mostrare quel continum della lingua poetica che si srotola secondo ritmo e cadenze preordinate. L'immagine è reperibile al link: http://www.stelle.com.br/pt/index_comedia.html (ultima consultazione 21.09.2021). 


\section{Durs Grünbein. Un'idea di Dante 3D \\ Rosalba Maletta}

\section{Bibliografia}

\section{Opere di Durs Grünbein}

Grünbein, Durs. Grauzone morgens. Gedichte (1985-1988). Suhrkamp, 1988.

—. Schädelbasislektion. Gedichte. Suhrkamp, 1991.

—. "Dieu trompe-l'œil." Schädelbasislektion, p. 19.

—. "Fisch im Medium". Schädelbasislektion, p. 20.

—. "Ultra Null." Schädelbasislektion, pp. 138-139.

—. Den Körper zerbrechen.Rede zur Entgegennahme des Georg-Büchner-Preises 1995. Mit der Laudatio "Portrait des Künstlers als junger Grenə̧hund" von Heiner Müller. Sonderdruck edition suhrkamp, 1995.

—. Galilei vermißt Dantes Hölle. Aufsätze 1989-1995. Suhrkamp, 1996.

—_. "Ameisenhafte Größe”. Galilei, cit., pp. 13-17 (Edition Text+Kritik, München 1990).

_. "Reflex und Exegese". Galilei, cit., pp. 61-66.

__. "Galilei vermißt Dantes Hölle und bleibt an den Maßen hängen." Galilei, cit., pp. 89104.

—_. "Mein babylonisches Hirn.”Galilei, pp. 18-33. (Die Schweizer Korrektur, Urs Engeler Editor, Basel 1995).

_. "Brief über den Sarkasmus und das Gedicht als Konzept" (Berlin, 20/9/91) Galilei, cit., pp. 50-54.

—. Nach den Satiren. Gedichte. Suhrkamp, 1999.

—. Das erste Jahr. Berliner Aufzeichnungen. Suhrkamp, 2000.

—. Warum schriftlos leben. Aufsätze. Suhrkamp, 2003.

__. "Warum schriftlos leben" (Mai 1999). Warum schriftlos leben. pp. 34-65.

—. Vom Schnee oder Descartes in Deutschland. Suhrkamp, 2003.

_. "Vom Schnee. Vorrede, Vorstudien, Nachträge". Sinn und Form. Beiträge zur Literatur, Heft $1 /$ 2004, Jahrgang 56, pp. 100-107. Ora anche in Aus der Traum (Kartei). Aufsätze und Notate. Suhrkamp, 2019, pp. 542-547 da cui citiamo.

—.Gedicht und Geheminis. Aufsätze 1990-2006. Suhrkamp, 2007.

—. "Zeit der Tiefseefische". Gedicht und Geheminis, cit., pp. 54-64.

_.."Das Gedicht und sein Geheimnis."Gedicht und Geheimnis, cit., pp. 84-94. (Erstdruck: Frau und Hund. Zeitschrift für kursives Denken, Heft 6, 2005, pp. 745-756).

—. Der cartesische Taucher. Drei Meditationen. Suhrkamp, 2008.

—. Die Bars von Atlantis. Eine Erkundung in vierzehn Tauchgängen. Suhrkamp, 2009.

—. "Kosmopolit". Die Bars von Atlantis, cit., pp. 31-33.

—. "Meerfahrt mit Dante." Die Bars von Atlantis, cit., pp. 37-44.

—_. "Dichtung als Schifffahrt." Die Bars von Atlantis, pp. 16-17.

—.Vom Stellenwert der Worte. Frankfurter Poetikvorlesung 2009. Sonderdruck Suhrkamp, 2010. 


\section{Durs Grünbein. Un'idea di Dante 3D \\ Rosalba Maletta}

—. Koloßim Nebel.Gedichte, Suhrkamp, Berlin 2012.

—. "Studien in Aquamarin." Koloß im Nebel, cit., pp. 102-110

—.Die Jahre im Zoo. Ein Kaleidoskop. Suhrkamp, 2015.

—. Zündkerzen. Gedichte. Suhrkamp, 2017.

—. Aus der Traum (Kartei). Aufsätze und Notate. Suhrkamp, 2019.

—. "Die Akademie des Meeres". Aus der Traum (Kartei), cit., pp. 75-89.

—. "Das Punktum des Gedichts". Aus der Traum (Kartei), cit., pp. 90-97.

—. "Sterne, Städte, Gehirne." Aus der Traum (Kartei), cit., pp. 417-439.

—. "Die Insel, die es nicht gibt". Aus der Traum (Kartei), cit., pp. 560-565.

- Jenseits der Literatur. Oxford Lectures. Suhrkamp, 2020.

—. Il bosco bianco. Poesie e altri scritti. Nota introduttiva di Elio Franzini, a cura di Rosalba Maletta. Mimesis, 2020.

—. "Ein flotter Sechser." Dante Alighieri, 1 Sonett 30 Übersetzungen, hrsg. von Judith Kasper, Andrea Renker, Fabien Vitali. turia+kant, 2021.

_. "Die unheimlichste Stelle." Serie "DANTES VERSE", hrsg. Von Jürgen Kaube. Frankfurter Allgemeine Zeitung aktualisiert am 03.07.2021-21:18: https://www.faz.net/aktuell/feuilleton/buecher/dantes-commedia/die-unheimlichstestelle-durs-gruenbein-ueber-dantes-verse-17418740.html.

- Europa, die Insel, die es nicht gibt. Allocuzione tenuta a Llubljana (Slovenia) alla fine di agosto del 2021, testo inedito, qui citato per gentile concessione dell'autore.

\section{Interviste e conversazioni}

Grünbein, Durs, und Heinz-Norbert Jocks. Durs Grünbein im Gespräch mit Heinz-Norbert Jocks. DuMont, 2001.

Grünbein, Durs, und Joachim Kaiser. Auf Tauchgang im Abendland. Edition Stiftung Schloss Neuhardenberg-Theater der Zeit, 2009.

Grünbein, Durs, und Michael Eskin. "Tauchen mit Descartes. Gespräch mit Durs Grünbein." Sinn und Form. Beiträge zur Literatur, hrsg. von der Akademie Der Künste, 63. Jahr, 3. Heft, Mai-Juni 2011, pp. 329-402.

Grünbein, Durs, und Silvia Ruzzenenti. "Dantes Stimme. Durs Grünbein im Gespräch mit Silvia Ruzzenenti". Deutsches Dante-Jahrbuch, n. 96, 2021, in corso di stampa.

\section{Registrazioni video}

31 ottobre 2020 ore 21.00 Durs Grünbein. Una conversazione con Dante condotta dal Professor Maurizio Pirro in sostituzione dell'Incontro di Monte Verità, causa emergenza sanitaria: http:/ /www.fondazioneteatro.ch/it/eventiletterari/services/event.html?evid=64ad5e28496c-4420-a17a-366740496c11.

27 febbraio 2014 ore 19.30 ICI BERLIN: Dicbtung als Wissen. Durs Grünbein spricht über Dante: https://www.ici-berlin.org/events/dichtung-als-wissen/. 


\section{Durs Grünbein. Un'idea di Dante 3D \\ Rosalba Maletta}

\section{Altre fonti bibliografiche}

Alighieri, Dante. La divina Commedia. Inferno - Purgatorio - Paradiso, 3 voll., a cura di Natalino Sapegno. La Nuova Italia, 1968 (sec. ediz. ricomposta).

—. Convivio, a cura di Giorgio Inglese. BUR, 20075.

—. Rime, a cura di Gianfranco Contini, con un saggio di Maurizio Perugi. Einaudi, 2021 (prima ed. 1939).

—. Vita Nuova. Con una guida alla lettura di Edoardo Sanguineti. Note di Alfonso Berardinelli, Garzanti $1982^{3}$.

Adolf-Altenberg, Gertrude. "La figura di Dante nei paesi germanici." Aevum, vol. 32, no. 5/6, 1958, pp. 517-536.

Auerbach, Erich. Mimesis. Il realismo nella letteratura occidentale, con un saggio introduttivo di Alberto Romagnoli, trad. Alberto Romagnoli e Hans Hinterhäuser, 2 voll. Einaudi, 1956.

_. Studi su Dante, trad. Maria Luisa de Pieri Bonino e Dante Della Terza. Feltrinelli, 1963.

Barolini, Teodolinda. La "Commedia" senza Dio. Dante e la creazione di una realtà virtuale, trad. Roberta Antognini. Feltrinelli, 2003.

—. Il miglior fabbro, trad. Paolo Barbera. Bollati Boringhieri, 1993.

- Il secolo di Dante. Viaggio alle origini della cultura letteraria italiana, trad. di Giuseppe Bernardi. Bompiani, 2012.

Barthes, Roland. Il brusio della lingua. Saggi critici IV. Einaudi, 1988.

Baudrillard, Jean. Simulacres et simulations. Galilée, 1981.

Bertolucci, Attilio. "La felicità in versi. Intervista di Giorgio Manacorda". La Repubblica 23 febbraio 1991. https://ricerca.repubblica.it/repubblica/archivio/repubblica/ 1991/02/23/attilio-bertolucci -la-felicita-in-versi.html.

Chantraine, Pierre. Dictionnaire étymologique de la langue grècque. Histoire des mots. Nouvelle édition avec supplement, Klincksieck, 1999.

Contini, Gianfranco. "Esercizio di interpretazione sopra un sonetto di Dante (1947)" con "Postilla del 1975". Un'idea di Dante, cit., pp. 20-31.

—. Un'idea di Dante. Saggi danteschi. Einaudi, $2001^{3}$.

—. "Un'interpretazione di Dante" (1965). Un'idea di Dante, cit., pp. 69-111.

Curtius, Ernst Robert. Letteratura della letteratura. Saggi critici, a cura di Lea Ritter Santini. Il Mulino, $1984^{3}$.

__. "La nave degli Argonauti.". Letteratura della letteratura., cit., pp. 301-325.

Eskin, Michael. "Descartes of Metaphor. On Grünbein's Vom Schnee." Leeder, Kareen J. (hrsg. von). Schaltstelle. Neue deutsche Lyrik im Dialog. Rodopi, 2007, pp. 163-179.

—. "The Driving Bell and the Bristlemouth. The Art of Grünbein's Prose." Grünbein, Durs. The Bars of Atlantis. Selected Essays, with an Introduction by Michael Eskin. Edited by Michael Eskin, translated from the German by John Crutchfield, Michael Hoffmann, Andrew Shields. Farrar, Straus and Giroux, 2010, pp. VII-XVIII.

—_. "Durs Grünbein and the European Tradition.” Durs Grünbein. A Companion, cit., pp. 23-38.

—. Eskin, Michael; Leeder, Karen ; Young, Cristopher (Eds.). Durs Grünbein. A Companion. Walter de Gruyter, 2013. 


\section{Durs Grünbein. Un'idea di Dante 3D \\ Rosalba Maletta}

—. "Stimmengewirr vieler Zeiten: Grünbein's Dialogue with Dante, Baudelaire, und Mandel'shtam." The Germanic Review, 77, 2002, pp. 34-51.

Freud, Sigmund. "Die Frage der Laienanalyse.” 1926. GW XIV, pp. 207-284.

—. Gesammelte Werke. Hrsg. v. Anna Freud et alii. 18 voll. Imago Publishing Co., 1940-1952. Fischer 1960-1968. (GW)

—. "Jenseits des Lustprinzips." 1920. GW XIII, pp. 1-69.

—. Traumdeutung. 1899. GW II-III.

—. "Das Unheimliche." 1919. GW XII, pp. 229-268.

Gross, Marek. Bruch und Erinnerung. Durs Grünbein's Poetik. LIT Verlag, 2011.

—. "In den Straßen Roms. Ein Gespräch mit Durs Grünbein". Anhang in Bruch und Erinnerung, cit., pp. 222-240.

Hofstadter, Douglas R. Le Ton Beau de Marot. In Praise of The Music of Language. Basic Books, 1997.

Krämer, Olav. "Bildliches Denken als Erkenntnismodus zwischen Poesie und Wissenschaft. Grünbein über Dante, Darwin, Hopkins und Goethe.” Bremer, Kai; Lampart, Fabian; Wesche, Jörg (hrsg. von). Schreiben am Schnittpunkt. Poesie und Wissen bei Durs Grünbein. Rombach Verlag, 2007, pp. 241-259.

Lacan, Jacques. Autres écrits, Seuil, 2001.

—. Écrits, Seuil, 1966.

—. "L'étourdit”. Scilicet 4, Seuil, 1973, pp. 5-52. Ora disponibile in Autres écrits, pp. 449-495.

_. "L'instance de la lettre dans l'inconscient ou la raison d'après Freud." 1957. Écrits, cit., Seuil Paris, 1966, pp. 493-528.

—. Le Séminaire. Livre XX. Encore (1972-73). Seuil, 1975.

—. Il Seminario. Libro X. L'angoscia 1962-1963, a cura di Antonio di Ciaccia. Einaudi, 2007.

Lampart, Fabian. "Jeder in seiner Welt, so viele Welten.... Durs Grünbeins Dante". Michale Braun; Heinz Ludiwig Arnold (hrsg. von). Dur Grünbein. Text + Kritik, 2002, pp. 49-59.

Maletta, Rosalba. “Grünbein e Dante. Sedendo et quiescendo anima efficitur sapiens". Perassi, Emilia (a cura di). Dante nelle Letterature straniere. Di/Segni. Dipartimento di Lingue e Letterature Straniere, Università degli Studi di Milano, Ledizoni, 2021, pp. 283-302.

_. "Poesia e discorso civile. Leggere la contemporaneità con Durs Grünbein". Grünbein, Durs. Il bosco bianco. Poesie e altri scritti, cit., pp. 59-99.

Manacorda, Giorgio. "Attilio Bertolucci. La felicità in versi. Intervista di Giorgio Manacorda”. https://ricerca.repubblica.it/repubblica/archivio/repubblica/1991/02/23/attiliobertolucci-la-felicita-in-versi.html.

Ossola, Carlo. Introdurione alla Divina Commedia. Marsilio, 2021.

Pascarelli, Pietro. "Il perturbante (Das Unheimliche) cent'anni dopo." European Journal of Psychoanalysis", 29.12.2019: https://www.journal-psychoanalysis.eu/il-perturbante-dasunheimliche-s-freud-1919-centanni-dopo/.

Patapievici, Horia-Roman. Gli occhi di Beatrice. Com'era davvero il mondo di Dante?, trad. Smaranda Bratu Elian. Bruno Mondadori $2006^{2}$. 


\section{Durs Grünbein. Un'idea di Dante 3D \\ Rosalba Maletta}

Penrose, Roger. Dal Big Bang all'eternità. I cicli temporali che danno forma all' universo. Rizzoli, Milano, 2011.

Petzholdt, Julius. Zur Geschichte der Danteausgaben von Pbilalethes, Druck von Johannes Pässler, Dresden 1884. https://archive.org/details/bub_gb_RTouAAAAYAAJ/page/n6/mode/ 1 up (ultima consultazione 22.09.2021).

Raimondi, Ezio. "Hermann Broch e la memoria della letteratura". La metamorfosi della parola. Da Dante a Montale, cit., pp. 13-72.

- La metamorfosi della parola. Da Dante a Montale, a cura di Jonathan Sisco, Bruno Mondadori, 2004.

Rancière, Jacques. Le partage du sensible. Esthétique et politique. La fabrique éditions. Paris 2000.

Rastier, François. “Le langage a-t-il une origine?”. Texto!, juillet 2007, vol. XII, n. 3, pp. 7-19.

Rilke, Rainer Maria. Werke. Kommentierte Ausgabe in 4 Bänden, hrsg. von Manfred Engel, Ulrich Fülleborn, Horst Nalewski, August Stahl. Insel, 1996.

Ruzzenenti Silvia. “Zu Durs Grünbeins lectura Dantis”. Michael Dallapiazza; Silvia Ruzzenenti (Hrsg.). Mittelalterbilder in der deutschsprachigen Literatur des langen 20. Jabrbunderts. Rezeption - Transfer - Transformation. Königshausen \& Neumann, 2018, pp. 187-215.

Singleton, Charles Southward. "Elementi di struttura". La poesia della Divina Commedia, cit., pp. 15-129.

—. "The irreducible Dove". Comparative Literature IX, (1957), pp. 129-135.

—. La poesia della Divina Commedia, Il Mulino, Bologna 2021(1978).

Unfer Lukoschik, Rita. "Rassegna della ricezione di Dante in area linguistica tedesca". Dante. Rivista Internazionale di Studi su Dante Aligbieri, n. 6, 2009, pp. 179-191.

Unfer Lukoschik, Rita e Michael Dallapiazza (a cura di). La Ricezione di Dante Alighieri. Impulsi e Tensioni. Atti Del Convegno Internazionale All'Università di Urbino 26 E 27 Maggio 2010. Peter Lang, 2011.

Waentig, Peter Wolfgang. "Giovanni di Sassonia. Il re dantista”. Atti Accademia Roveretana degli Agiati, a cura di Giuseppe Osti, Rovereto, Accademia Roveretana degli Agiati, 2010, pp. 311-335.

Dizionari online

DUDEN: https://www.duden.de

GARZANTI: https://www.garzantilinguistica.it

DER DIGITALE GRIMM: https://woerterbuchnetz.de/?sigle=DWB\#0

TRECCANI VOCABOLARIO: https://www.treccani.it/vocabolario

\section{Sitografia}

MKRL $A B$ | Ideas Factory: https://www.mkrlab.eu

MONACO NATURE ENCYCLOPEDIA: https:/ / wnw. monaconatureencyclopedia.com

DANTE ONLINE: Consulenza scientifica Società Dantesca Italiana: http://www.danteonline.it/italiano/opere_indice.htm 